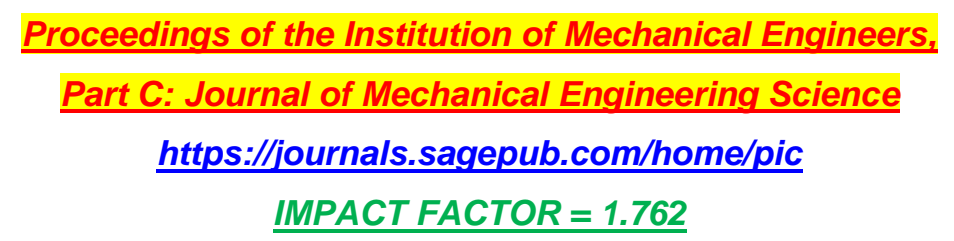

Accepted $4^{\text {th }}$ February 2022

\title{
COMPUTATION OF THERMO-SOLUTAL CONVECTION WITH SORET-DUFOUR CROSS DIFFUSION IN A VERTICAL DUCT CONTAINING CARBON/METALLIC NANOFLUIDS

\author{
J.C. Umavathi ${ }^{1 *}$ and O. Anwar Bég ${ }^{2}$ \\ ${ }^{1}$ Professor of Applied Mathematics, Department of Mathematics, Gulbarga University, Gulbarga-585 106, \\ Karnataka, India. Email: drumavathi@ rediffmail.com
}

\begin{abstract}
${ }^{2}$ Professor of Engineering Science \& Director-Multi-Physical Engineering Sciences Group, Mechanical Engineering Department, School of Science, Engineering and Environment (SEE), University of Salford, Manchester, M54WT, UK. Email: $\underline{\text { O.A.Beg@salford.ac.uk/gortoab@gmail.com }}$
\end{abstract}

\begin{abstract}
Duct flows constitute an important category of modern thermal engineering. Optimizing efficiency has become a significant objective in the $21^{\text {st }}$ century in, for example, heating ventilation and air-conditioning (HVAC), coolant or heat transfer fluid flows in a nuclear power reactor, heat exchanger design etc, and this has been achieved by either new materials (improved thermal insulation properties) constituting the duct walls, novel geometric designs or improved working fluids. Nanotechnology has infiltrated into duct design in parallel with many other fields of mechanical, medical and energy engineering. Motivated by the excellent potential of nanofluids, a subset of materials engineered at the nanoscale, in the present work, a new mathematical model is developed for natural convection in a rectangular vertical duct containing nanofluid. The left and right walls of the duct are maintained at constant and unequal temperatures, while the front and rear walls of the duct are insulated. Thermo-solutal (double-diffusive) natural convection of aqueous nanofluid containing various metallic nanoparticles (e. g. copper, titanium oxide) or carbon-based nanoparticles (e. g. diamond, silicon oxide) is simulated. The Tiwari-Das nanoscale volume fraction model is used in addition to the Brinkman and Maxwell models for defining the properties of the nanofluid. The partial differential conservation equations for mass, momentum and energy are non-dimensionalized via appropriate transformations and the resulting boundary value problem is solved with a second-order accurate implicit finite difference technique employing Southwell-Over-Relaxation (SOR). Mesh independence tests are conducted. Extensive visualization of the solutions for velocity, temperature, nanoparticle concentration (volume fraction) are presented for five different nanoparticles (silicon oxide, diamond, copper, titanium oxide and silver), thermal Grashof number, nanoparticle species (solutal) Grashof number, volume fraction of nanoparticles (i.e. percentage doping), Dufour number, Soret number, Prandtl number, Schmidt number and duct aspect ratio. It is observed that the heat transfer rate (Nusselt number) at both the walls is maximized for diamond nanoparticles and minimized for silicon oxide nanoparticles. Further the heat transfer rate for clear fluid is lower when compared with nanofluid, confirming that nanoparticles achieve the desired thermal enhancement at the boundaries also. The mass transfer at both walls (Sherwood number) however is not significantly influenced by any particular type of nanoparticle, thermal and concentration Grashof number and is depleted with higher values of Dufour, Prandtl, Soret and Schmidt numbers in addition to aspect ratio. However, Sherwood numbers at both the left and right duct walls are substantially boosted with greater solid volume fraction of nanoparticles.
\end{abstract}

*Corresponding author

KEYWORDS: Finite difference method (FDM), vertical duct, Tiwari-Das model; Brinkman model; Maxwell model; nanofluid volume fraction; thermal process technologies. 


\section{NOMENCLATURE}

A

a

$b$

C

$c$

$C_{S}$

$C_{P}$

D

$D f$

$g$

$G r_{T}$

$G r_{C}$

K

$K_{\text {Tf }}$

$P$

Pr

Sc

Sr

$T$

$T_{0}$

$U, V, W \quad$ dimensional components of velocity $(\mathrm{m} / \mathrm{s})$

$u, v, w$

$X, Y, Z$

duct aspect ratio (length to width ratio) $(a / b)(-)$

length of the duct $(\mathrm{m})$

breadth of the duct $(\mathrm{m})$

dimensional concentration (Moles $/ \mathrm{m}^{3}$ )

concentration in dimensionless form (-)

concentration susceptibility (Moles $/ \mathrm{m}^{3}$ )

specific heat capacity (isobaric) $(\mathrm{J} / \mathrm{kgK})$

nanoparticle solutal diffusivity $\left(\mathrm{m}^{2} / \mathrm{s}\right)$

Dufour parameter $\left(\left(\frac{D}{v}\right)_{f} \frac{K_{T f}\left(C_{2}-C_{1}\right)}{C_{S} C_{P}\left(T_{2}-T_{1}\right)}\right)(-)$

gravitational acceleration $\left(\mathrm{m} / \mathrm{s}^{2}\right)$

thermal Grashof number $\left(g \beta_{f} \Delta T b^{3} \rho_{f}^{2} / \mu_{f}^{2}\right)(-)$

solute Grashof number $\left(g \beta_{f} \Delta C b^{3} \rho_{f}^{2} / \mu_{f}^{2}\right)(-)$

conductivity of the fluid (W/mK)

thermal diffusion ratio (-)

pressure $(\mathrm{Pa})$

Prandtl number $\left(\frac{v}{\alpha}\right)_{f}(-)$

Schmidt number $\left(\frac{v}{D}\right)_{f}(-)$

Soret number $\left(\left(\frac{D}{v}\right)_{f} \frac{K_{T f}\left(C_{2}-C_{1}\right)}{T_{m}\left(T_{2}-T_{1}\right)}\right)(-)$

temperature $(\mathrm{K})$

reference temperature $(\mathrm{K})$

dimensionless velocity components (-)

dimensional coordinates of space (m) 
$x, y, z \quad$ dimensionless space coordinates (-)

\section{Greek symbols}

$\begin{array}{ll}\alpha & \text { thermal diffusivity }\left(\mathrm{m}^{2} / \mathrm{s}\right) \\ \beta & \text { coefficient of thermal expansion }(/ \mathrm{K}) \\ \rho & \text { density }\left(\mathrm{kg} / \mathrm{m}^{3}\right) \\ \mu & \text { dynamic viscosity }(\mathrm{kg} / \mathrm{ms}) \\ v & \text { kinematic viscosity }\left(\mathrm{m}^{2} / \mathrm{s}\right) \\ \phi & \text { solid volume fraction i.e. dimensionless nanoparticle concentration }(\%) \\ \theta & \text { dimensionless temperature }(-)\end{array}$

\section{Subscripts}

1

left wall

2

right wall

$n f$

nanofluid

f

base fluid

$m$

mean

$s$

solid particles

\section{INTRODUCTION}

Free convection (flow driven by buoyancy forces) in ducts features in numerous diverse areas of modern technology including nuclear power plants, solar energy, electronics cooling, architectural fluid dynamics (fire plumes, thermal stacks, natural ventilation etc) and geothermics ${ }^{1,2}$. In building services and nuclear systems, conventional coolants possess low thermal conductivity which is a limitation in achieving optimal heat transfer. This limitation may be successfully overcome by using nanofluids which are nanoscale-engineered colloidal suspensions exhibiting thermal conductivity higher than that of conventional base fluids (air, water etc). The initial success of nanofluids in aerospace and automotive applications (radiator and jet engine thermal management) have stimulated intense activity in the research community exploring the 
deployment of these nanomaterials in other branches of science ${ }^{3}$. The heat transfer carrier fluids used are oil, ethanol, water and ethylene glycol. Choi and Eastman ${ }^{4}$ were the first engineers worldwide to demonstrate the efficacy of nanofluids, initiating this branch of modern fluid dynamics at the Argonne Energy Laboratory, Illinois, USA in the 1990s. A nanofluid contains dispersed nanoparticles which have high thermal conductivity. The conductivity of energy ${ }^{7}$, diffusivity of heat ${ }^{6}$, conductivity occurring due to electricity ${ }^{7}$ are all demonstrably improved using nanofluids. Adding metallic nanoparticles $^{8}$, metallic oxide nanoparticles ${ }^{9}$ and ceramic (carbon-based) nanoparticles ${ }^{10}$ in a conventional base fluid substantially improves the energy properties.

The performance of nanofluids can be robustly simulated by evaluating the viscosity and thermal conductivity ${ }^{11}$. The theories developed by Einstein ${ }^{12}$ and Brinkman ${ }^{13}$ to anticipate the effective viscosity, were under-estimated by experiments ${ }^{14}$. Hence more refined mathematical models were developed to compute the effective thermal conductivity and viscosity ${ }^{15}$ of fluids containing doped suspensions. The correlations based on the data extracted from experiments were elegantly derived by Corcione ${ }^{16}$ who included volume fraction, nanoparticle diameter and temperature.

Two main models were developed for nanofluid dynamic analysis - single-phase and two-phase. In the single-phase model ${ }^{17}$, the transport equations for the pure fluid are extended directly to those for nanofluids. The fluid and the suspended particles are assumed to be in a state of thermal equilibrium and both the nanoparticles and the base fluid flow with the same velocity (i. e. slip effects are negated). Tiwari and Das ${ }^{17}$ advocated that the nanofluid behaves more like a fluid rather than a mixture of nanoparticles and base fluid. Therefore, their modified single-phase model is more suitable than the two-phase approach for computational nanofluid mechanics. Buongiorno $^{18}$ claimed that the heat transfer intensification occurring due to the addition of nanoparticles is negligible. Jou and Tzeng ${ }^{19}$ further argue that there are thus far no universally approved theoretical studies and experimental data for the suitable choice of nanofluid dynamic models. A number of studies have successfully implemented the single-phase model including Umavathi and Shekar $^{20}$ who examined hydromagnetic Jeffery-Hamel flow of nanofluids between non-parallel duct flows. Umavathi and Monica $^{21}$ employed a Galerkin method to simulate the stationary and oscillatory 
convection in hydrodynamic instability of a porous medium regime saturated with viscoelastic nanofluid. Selimefendigil and Öztop ${ }^{22}$ studied numerically the flow separation effects on the power generation and conversion efficiency of a thermoelectric generator integrated channel flow with area expansion. They observed that the vortex behind the step extended over the thermoelectric device surface at the highest Reynolds number while vortices were also established behind the eccentric cone. Selimefendigil and Öztop ${ }^{23}$ examined the effects of using a partly curved porous layer on the thermal management and entropy generation features in a ventilated cavity filled with hybrid nanofluid under the effects of inclined magnetic field with a finite volume method. They noted that the porous layer height reduced the entropy generation in the domain above it and achieved the highest contribution to the overall entropy generation.

Heat transfer in different enclosure geometries filled with nanofluids have also motivated some attention (Fontes et al. ${ }^{24}$ and Kalidasan et al. ${ }^{25}$ ). Umavathi et al. ${ }^{26}$ explored numerically the characteristics of different metallic/carbon nanofluids inside a duct with one wall cooled by a constant temperature and the other heated by a constant temperature and the remaining walls thermally insulated. They observed that different Nusselt numbers are achieved with different types of nanoparticle e. g. silver, copper, diamond and titanium oxide although there is an optimum solid volume fraction which maximizes the heat transfer. Shekar and Umavathi ${ }^{27}$ studied viscous dissipation effects on non-Darcy mixed convection flow of nanofluid in a vertical channel. Hatami and Safari ${ }^{28}$ and Al-Weheibi et al. ${ }^{29}$ computed free convection enhancement in a square enclosure filled with nanofluid. Khanafer et al. ${ }^{30}$ scrutinized the behavior of nanofluid enclosed in a differentially heated cavity with Buongiorno's nanoscale model. They concluded that employing $\mathrm{Al}_{2} \mathrm{O}_{3}$-water nanofluid optimizes energy transfer at high volume fractions and buoyancy force magnitudes. Ho et al. ${ }^{31}$ and Santra et al. ${ }^{32}$ numerically investigated the properties of thermal convection in a square enclosure filled with nanofluid. Santra et al. ${ }^{32}$ applied the Ostwald-de Waele model for a non-Newtonian shear-thinning fluid. A novel thermal flow system configuration was investigated by Selimefendigil and Öztop ${ }^{33}$ with rotating cylinders and nanofluids in channel to increase the powers generated in the TEG module mounted between the channels. Inclusion of nanoparticles was found to be efficient in the TEG power enhancement when performance of the system was low. Thus, 
slight improvement was achieved with nanoparticles when a system with five cylinders at the highest speed and highest Reynolds number was considered. Assessment of a thermoelectric generator module located in between two channels where carbonnanotube/water nanofluid streams flow was studied with the collective effects of nanoparticle inclusion and flow pulsations by Selimefendigil and Öztop ${ }^{34}$. They observed that flow pulsation changes the dynamic features of thermoelectric power generated in the device. It was also observed that $24.4 \%$ enhancement in the power were achieved for nanofluid with flow pulsation when lowest and highest pulsation amplitudes were compared. Selimefendigil and Öztop ${ }^{35}$ further discussed the forced convection of pulsating nanofluid flow over corrugated parallel plate in the presence of inclined magnetic field. They concluded that via the inclusion of nanoparticles in pulsating flow there is a shift in the spatial average Nusselt number plots as compared to base fluid.

Umavathi et al..$^{36}$ analysed numerically the natural convective flow of nanofluids in a vertical rectangular porous medium duct using the Darcy-Forchheimer-Brinkman model. Umavathi ${ }^{37}$ also investigated the Rayleigh-Benard convection with periodic wall temperature effects in a nanofluid saturated porous medium layer. Diglio et al. ${ }^{38}$ studied analytically and experimentally the behavior of nanoparticles in fluid flow in heat exchangers in boreholes. Alsabery et $\mathrm{al}^{39}$ concentrated on the transient entropy generation and mixed convection due to a rotating hot inner cylinder within a square cavity having a flexible side wall by using the finite element method and arbitrary Lagrangian-Eulerian formulation. It was observed that various complex shaped wall deformations were established depending on the non-dimensional elastic modulus of the flexible right wall and cylinder rotation direction. Bhatti and Abdelsalam ${ }^{40}$ studied the peristaltically driven motion of Carreau fluid in a symmetric channel under the influence of both induced and applied magnetic fields. They showed that Tantalum and gold nanoparticles enhanced the temperature distribution. The bioconvection flow in Oldroyd-B nanofluid transport from a convectively heated surface was studied by Khan et $\mathrm{al}^{41}$. They noted that variation in relaxation time constant increased the velocity profile which was more progressive in the presence of slip effects. The presence of slip factor also improved the heat and mass transportation significantly. Bhatti et al. ${ }^{42}$ investigated the unsteady bioconvection flow confined between parallel rotating circular plates in a porous medium saturated with 
Williamson elastico-viscous nanofluid. They found that the motile microorganism profile was reduced when the Péclet number was increased, whereas the microorganism profile was increased when the bioconvection Schmidt number was increased.

Free convection in fluids occurs only by density variations. However, in doublediffusive convection, a distinct rate of diffusion is associated with distinct density gradients $^{43}$. In nanofluids the density variations occur by temperature gradient or by the gradients in the solid volume fraction. In such systems cross diffusion effects may also be prominent as noted by Gebhart ${ }^{44}$. Cross diffusion has been extensively studied by many researchers. The Dufour effect relates to the energy flux due to a species concentration gradient occurring and is the reciprocal phenomenon to the Soret effect which is associated with the mass (species) flux due to a temperature gradient, as noted by Bég et al. $^{45}$. Both effects can exert considerable influence on heat, mass and momentum characteristics in duct flows ${ }^{46}$. Cross diffusion and viscosity variation effects were addressed by Umavathi and Mohite $^{47}$ again for thermosolutal convection through nanofluid-saturated porous layers. Bhatti et al. ${ }^{48}$ computed the hydrodynamic/thermal slip effects on radiative hydromagnetic $\mathrm{Fe}_{3} \mathrm{O}_{4}$-water-based nanofluid flow from a nonlinear stretching sheet in Darcian porous media with cross diffusion effects. Garvandha et al. ${ }^{49}$ analyzed the phase change in nanofluid coating flow from an inclined stretching cylinder cross diffusion and curvature effects using MATLAB quadrature. Umavathi et al. ${ }^{50}$ computed the hydrodynamic stability in a micropolar nanofluidsaturated permeable medium. Pakravan and Yaghoubi ${ }^{51}$ investigated analytically the collective effects of Dufour diffusion-thermo gradient, Brownian motion and thermophoresis on free convection in nanofluids, achieving good correlation with experimental results.

Technical literature describing the deployment of nanofluids in duct flows to estimate the convective transport for two diffusing components is however limited. This has prompted the present investigation in which the deployment of metallic/carbon-based aqueous nanofluids in a vertical duct with thermo-solutal convection and cross diffusion effects is considered for the first time. All these aspects constitute the novelty of the present article. Simulations have been performed using different nanoparticles suspended in water for a range of parametric values representative of actual industrial thermal duct 
systems $^{52}$. The present work generalizes the study $i^{26}$ to consider Soret and Dufour effects and has to the authors' knowledge not been communicated thus far in the literature.

\section{MATHEMATICAL FORMULATION}

The duct regime under investigation is visualized in Figure 1. The left wall is sustained at constant temperature $T_{1}$ and the right wall is also isothermal and maintains a constant temperature $T_{2}$. The nanoparticle species concentration at the left wall is less that at the right wall $\left(C_{2}>C_{1}\right)$. The duct is filled with water-based nanofluid containing different nanoparticles. Soret (thermo-diffusion) and Dufour (diffuso-thermal) effects are considered. The nanofluid is treated as incompressible and the upward-directed flow is assumed to be hydrodynamically fully developed, steady and laminar. Both base fluid (water) and nanoparticles (metallic or carbon based) are in thermal equilibrium and no slip occurs between them. The viscosity and temperature are treated as constant and the Boussinesq approximation is assumed. Flow occurs by only under the action of thermal and solutal (nanoparticle species) buoyancy forces i. e. pure natural convection currents are mobilized. The following assumptions are therefore invoked:

$U=V=0, \quad \frac{\partial U}{\partial X}=\frac{\partial U}{\partial Y}=\frac{\partial V}{\partial X}=\frac{\partial V}{\partial Y}=0, \quad \frac{\partial P}{\partial X}=\frac{\partial P}{\partial Y}=\frac{\partial P}{\partial Z}=0$

Eqn. (1) is valid by virtue of fully developed flow and furthermore $\partial W / \partial Z=0$. Therefore, only the axial velocity $(W)$ is invoked but is independent of the Z- coordinate. The Navier-Stokes viscous flow (momentum conservation), energy conservation and nanoparticle species conservation equations (based on Newton's law of viscosity, Fourier's law of heat conduction and Fick's law of species diffusion), may therefore be reduced to the following forms: (following Umavathi et al. ${ }^{26}$ and Bég et al. ${ }^{45}$ )

$\mu_{n f}\left(\frac{\partial^{2} W}{\partial X^{2}}+\frac{\partial^{2} W}{\partial Y^{2}}\right)+g(\rho \beta)_{n f}\left(\left(T-T_{0}\right)-\left(C-C_{0}\right)\right)=0$. 
$\frac{\partial^{2} T}{\partial X^{2}}+\frac{\partial^{2} T}{\partial Y^{2}}+\frac{D K_{T f}}{C_{s} C_{p} \alpha_{n f}}\left(\frac{\partial^{2} C}{\partial X^{2}}+\frac{\partial^{2} C}{\partial Y^{2}}\right)=0$.

$\frac{\partial^{2} C}{\partial X^{2}}+\frac{\partial^{2} C}{\partial Y^{2}}+\frac{K_{T f}}{T_{m}}\left(\frac{\partial^{2} T}{\partial X^{2}}+\frac{\partial^{2} T}{\partial Y^{2}}\right)=0$.

Thermal dispersion, stratification and relaxation effects are ignored. Equations (2) and (3) are solved subject to the following boundary conditions:

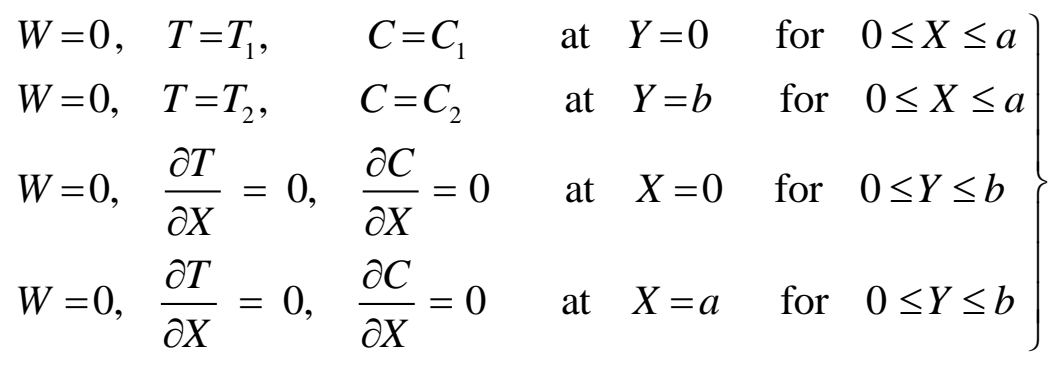

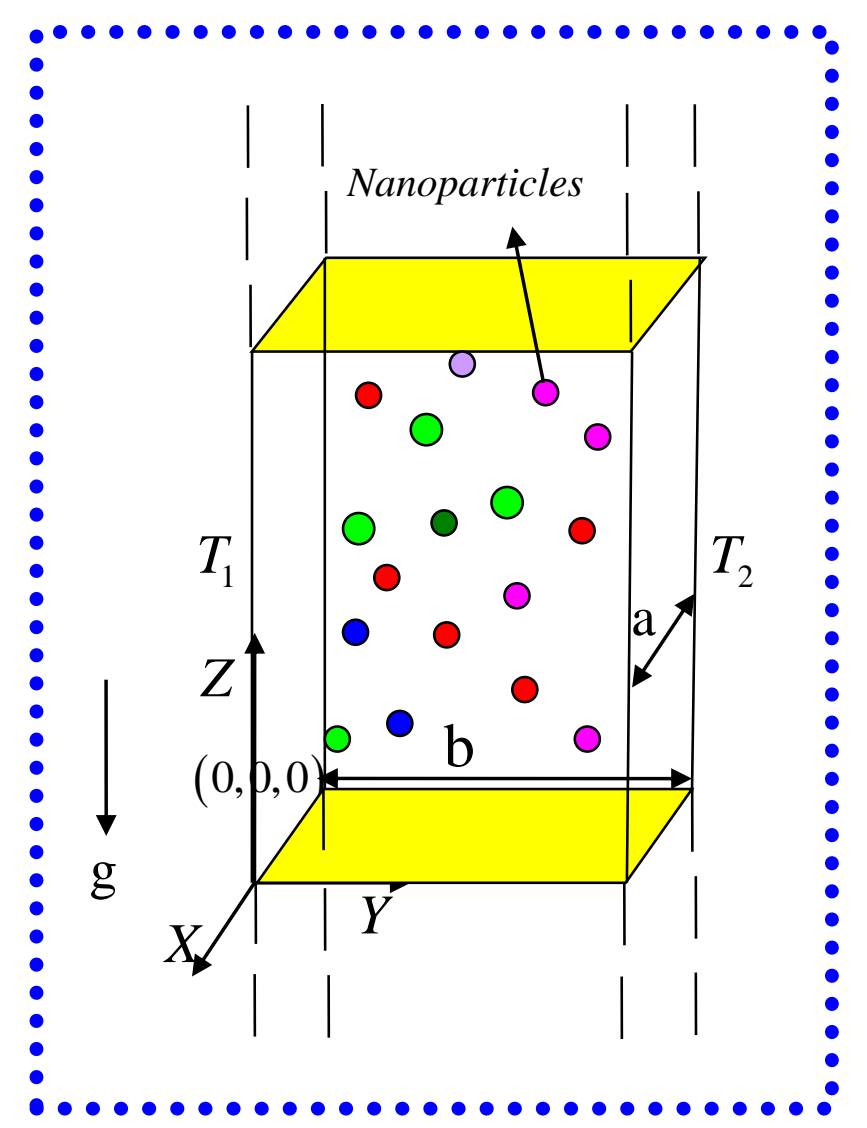

Fig. 1. Physical configuration for double-diffusive nanofluid convection in a vertical duct. 
The correlations for the density, thermal expansion coefficient, thermal diffusivity, heat capacitance and viscosity for the nanofluid $\left(\right.$ Brinkman $\left.^{13}\right)$ are adopted as:

$(\rho)_{n f}=(1-\phi) \rho_{f}+\phi \rho_{s}$.

$(\rho \beta)_{n f}=(1-\phi)(\rho \beta)_{f}+\phi(\rho \beta)_{s}$.

$\alpha_{n f}=\frac{K_{n f}}{\left(\rho C_{p}\right)_{n f}}$.

$\left(\rho C_{p}\right)_{n f}=(1-\phi)\left(\rho C_{p}\right)_{f}+\phi\left(\rho C_{p}\right)_{s}$

$\mu_{n f}=\frac{\mu_{f}}{(1-\phi)^{2.5}}$

The correlation for thermal diffusion is considered as in Maxwell ${ }^{53}$

$$
K_{n f}=K_{f}\left(\frac{K_{s}+2 K_{f}-2 \phi\left(K_{f}-K_{s}\right)}{K_{s}+2 K_{f}+\phi\left(K_{f}-K_{s}\right)}\right)
$$

Implementing the following dimensionless variables:

$$
\begin{aligned}
& x=\frac{X}{b}, \quad y=\frac{Y}{b}, \quad w=\frac{W \rho_{f} b}{\mu_{f}}, \quad \theta=\frac{\left(T-T_{0}\right)}{\left(T_{2}-T_{1}\right)}, \quad c=\frac{\left(C-C_{0}\right)}{\left(C_{2}-C_{1}\right)}, \quad T_{0}=\frac{\left(T_{1}+T_{2}\right)}{2}, \\
& C_{0}=\frac{\left(C_{1}+C_{2}\right)}{2}, \quad G r_{T}=\frac{g \beta_{f}\left(T_{2}-T_{1}\right) b^{3} \rho_{f}^{2}}{\mu_{f}^{2}}, \quad G r_{C}=\frac{g \beta_{f}\left(C_{2}-C_{1}\right) b^{3} \rho_{f}^{2}}{\mu_{f}^{2}}, \\
& D f=\left(\frac{D}{v}\right)_{f} \frac{K_{T f}\left(C_{2}-C_{1}\right)}{C_{S} C_{P}\left(T_{2}-T_{1}\right)}, \quad \operatorname{Pr}=\left(\frac{v}{\alpha}\right)_{f}, \quad S r=\left(\frac{D}{v}\right)_{f} \frac{K_{T f}\left(C_{2}-C_{1}\right)}{T_{m}\left(T_{2}-T_{1}\right)}, \quad S c=\left(\frac{v}{D}\right)_{f} .
\end{aligned}
$$

The governing Eqns. (2) to (4) are thereby reduced to: 


$$
\left.\begin{array}{l}
\frac{\partial^{2} w}{\partial x^{2}}+\frac{\partial^{2} w}{\partial y^{2}}+G r_{T}(1-\phi)^{2.5}\left(1-\phi+\phi \frac{(\rho \beta)_{s}}{(\rho \beta)_{f}}\right) \theta- \\
G r_{C}(1-\phi)^{2.5}\left(1-\phi+\phi \frac{(\rho \beta)_{s}}{(\rho \beta)_{f}}\right) c=0 . \\
\frac{\partial^{2} \theta}{\partial x^{2}}+\frac{\partial^{2} \theta}{\partial y^{2}}+\frac{D f \operatorname{Pr} K_{f}\left(\rho C_{p}\right)_{n f}}{K_{n f}\left(\rho C_{p}\right)_{f}}\left(\frac{\partial^{2} c}{\partial x^{2}}+\frac{\partial^{2} c}{\partial y^{2}}\right)=0 . \\
\frac{\partial^{2} c}{\partial x^{2}}+\frac{\partial^{2} c}{\partial y^{2}}+\operatorname{Sr} S c\left(\frac{\partial^{2} \theta}{\partial x^{2}}+\frac{\partial^{2} \theta}{\partial y^{2}}\right)=0 .
\end{array}\right\}
$$

The respective boundary conditions after inserting Eq. (12) emerge as:

$w=0, \quad \theta=-0.5, \quad c=-0.5 \quad$ at $\quad y=0 \quad$ for $0 \leq x \leq A$

$w=0, \quad \theta=0.5, \quad c=0.5 \quad$ at $\quad y=1 \quad$ for $0 \leq x \leq A$

$w=0, \quad \frac{\partial \theta}{\partial x}=0, \quad \frac{\partial c}{\partial x}=0 \quad$ at $\quad x=0$ and $x=A$ for $0 \leq y \leq 1$

The volumetric flow rate, skin friction, Nusselt number and Sherwood number, are estimated and shown in Tables 2 to 4 using water as a carrier fluid.

\section{COMPUTATIONAL PROCEDURE}

The system of governing Eqns. (13) to (15), in conjunction with the boundary conditions as defined in Eqn. (16), is solved with a robust finite difference method. Uniform structured grids are used for the discretization of the computational domain. Second order accuracy of the central difference is employed, and the following difference equations are obtained.

$$
\left.\begin{array}{l}
\frac{w_{i+1, j}-2 w_{i, j}+w_{i-1, j}}{(\Delta x)^{2}}+\frac{w_{i, j+1}-2 w_{i, j}+w_{i, j-1}}{(\Delta y)^{2}}+G r_{T}(1-\phi)^{2.5}\left(1-\phi+\phi \frac{(\rho \beta)_{s}}{(\rho \beta)_{f}}\right) \theta_{i, j}- \\
G r_{C}(1-\phi)^{2.5}\left(\phi \frac{(\rho \beta)_{s}}{(\rho \beta)_{f}}+1-\phi\right) c_{i, j}=0 .
\end{array}\right\}
$$




$$
\left.\begin{array}{l}
\frac{\theta_{i-1, j}-2 \theta_{i, j}+\theta_{i+1, j}}{(\Delta x)^{2}}+\frac{\theta_{i, j-1}-2 \theta_{i, j}+\theta_{i, j+1}}{(\Delta y)^{2}}+ \\
\frac{D f \operatorname{Pr} K_{f}\left(\rho C_{p}\right)_{n f}}{K_{n f}\left(\rho C_{p}\right)_{f}}\left(\frac{c_{i+1, j}-2 c_{i, j}+c_{i-1, j}}{(\Delta x)^{2}}+\frac{c_{i, j+1}-2 c_{i, j}+c_{i, j-1}}{(\Delta y)^{2}}\right)=0 . \\
\frac{c_{i+1, j}-2 c_{i, j}+c_{i-1, j}}{(\Delta x)^{2}}+\frac{c_{i, j+1}-2 c_{i, j}+c_{i, j-1}}{(\Delta y)^{2}}+ \\
\operatorname{Sr} \operatorname{Sc}\left(\frac{\theta_{i+1, j}-2 \theta_{i, j}+\theta_{i-1, j}}{(\Delta x)^{2}}+\frac{\theta_{i, j+1}-2 \theta_{i, j}+\theta_{i, j-1}}{(\Delta y)^{2}}\right)=0 .
\end{array}\right\}
$$

The boundary conditions are obtained as follows:

$$
\left.\begin{array}{l}
w_{i, 0}=-w_{i, 1}, \theta_{i, 0}=-1-\theta_{i, 1}, \quad c_{i, 0}=-1-c_{i, 1} \\
w_{i, N y+1}=-w_{i, N y}, \theta_{i, N y+1}=1-\theta_{i, N y}, \quad c_{i, N y+1}=1-c_{i, N y} \\
w_{0, j}=-w_{1, j}, \theta_{0, j}=\theta_{1, j}, \quad c_{0, j}=c_{1, j} \\
w_{N x+1, j}=-w_{N x, j}, \theta_{N x+1, j}=\theta_{N x, j}, \quad c_{N x+1, j}=c_{N x, j}
\end{array}\right\}
$$

Here 1 to $N x$ is the range of $i$ and 1 to $N y$ is the range of $j$. Starting from the assigned initial fields of the dependent variables, the discretized algebraic governing equations are solved iteratively. A standard Southwell-Over-Relaxation technique is incorporated for the convergence. The solutions for the velocity, temperature and nanoparticle concentration fields are accepted as converged when the maximum absolute value of the velocity, temperature and nanoparticle concentration at any grid-node between two consecutive iterations, are smaller than the prescribed tolerance values. The numerical method is validated by a grid-independence study. The Nusselt number (rate of heat transfer) and skin friction at the left and right walls of the duct are evaluated using $10 \mathrm{X}$ 10, 50 X 50, 100 X 100, 150 X 150 and 200 X 200 grids and are shown in Table-1. The solutions for $50 \times 50,100 \times 100,150 \times 150$ and $200 \times 200$ grids are quite similar. Hence 100 points along the $y$-direction and $100 \times A$ points along the $x$-direction are selected for the calculations. The iterations are carried out and convergence when the solutions on the grids are less than $10^{-14}$. Further details of the numerical method are available in Hoffman $^{54}$. Further the present code is validated with Umavathi et $\mathrm{al}^{26}$. In the absence of 
Dufour, Soret and Schmidt parameters the model reduces exactly to that studied by Umavathi et $\mathrm{al}^{26}$ when $\mathrm{Br}=0$.

Table 1. Grid independence analysis

\begin{tabular}{|c|c|c|c|c|}
\hline Grids & $\left(\frac{d \theta}{d y}\right)_{y=0}$ & $\left(\frac{d \theta}{d y}\right)_{y=1}$ & $\left(\frac{d w}{d y}\right)_{y=0}$ & $\left(\frac{d w}{d y}\right)_{y=1}$ \\
\hline $10 \mathrm{X} 10$ & 0.578568077182 & 0.578568077183 & -0.136563390615 & -0.136559764692 \\
\hline $50 \mathrm{X} 50$ & 0.578574796487 & 0.578574796504 & -0.138822176467 & -0.138797452475 \\
\hline $100 \mathrm{X} 100$ & 0.578583194869 & 0.578583194941 & -0.138906143237 & -0.138854866430 \\
\hline $150 \mathrm{X} 150$ & 0.578591592422 & 0.578591592582 & -0.138932521060 & -0.138854677778 \\
\hline 200X200 & 0.578599989146 & 0.578599989424 & -0.138950390438 & -0.138845977257 \\
\hline
\end{tabular}

\section{RESULTS}

Extensive graphical plots for the impact of all key parameters are shown in Figs. 2-7 on momentum, thermal and nanoparticle mass transfer characteristics in carbon/metallic nanofluid flowing in the vertical duct. The results of this study are presented in terms of the effects of different nanoparticles (silver, diamond, copper, $\mathrm{TiO}_{2}$ and $\mathrm{SiO}_{2}$ ), the thermal Grashof number $\left(1 \leq G r_{T} \leq 20\right)$, concentration Grashof number $\left(1 \leq G r_{C} \leq 15\right)$, solid volume fraction $(0 \leq \phi \leq 0.05)$, Dufour parameter $(0 \leq D f \leq 1)$ and aspect ratio $(0.5 \leq A \leq 2.0)$ on the velocity, temperature and nanoparticle concentration profiles and these are visualized in two different forms. The graphs are pictured in the threedimensional form to provide a perspective of the direction of the flow in the duct. The graphs are also drawn in one dimension by fixing the values of $y=0.5$ for the values of $x$ from 0 to 1 which visualizes in a better way the effects of the controlling parameters on the flow. 

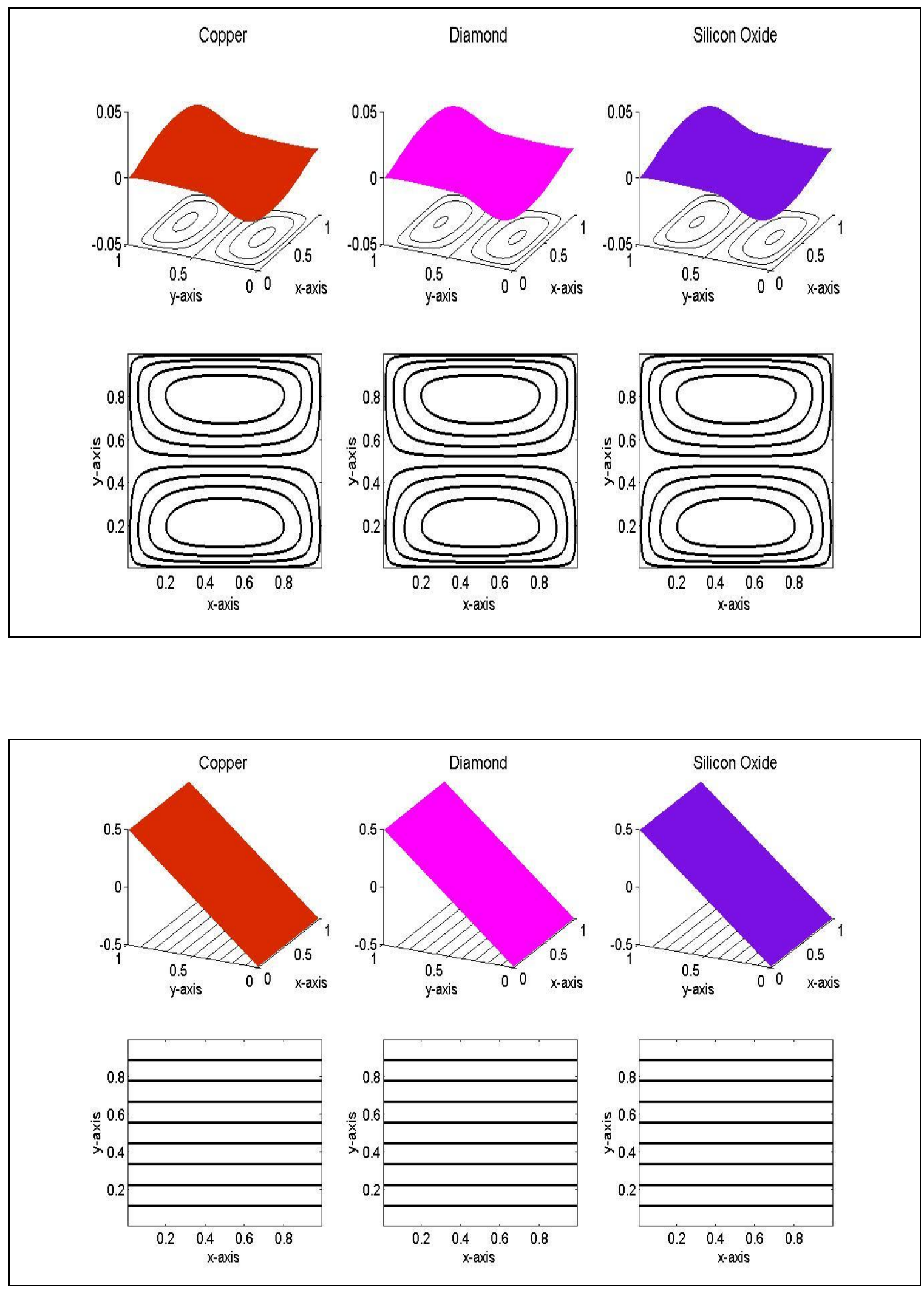


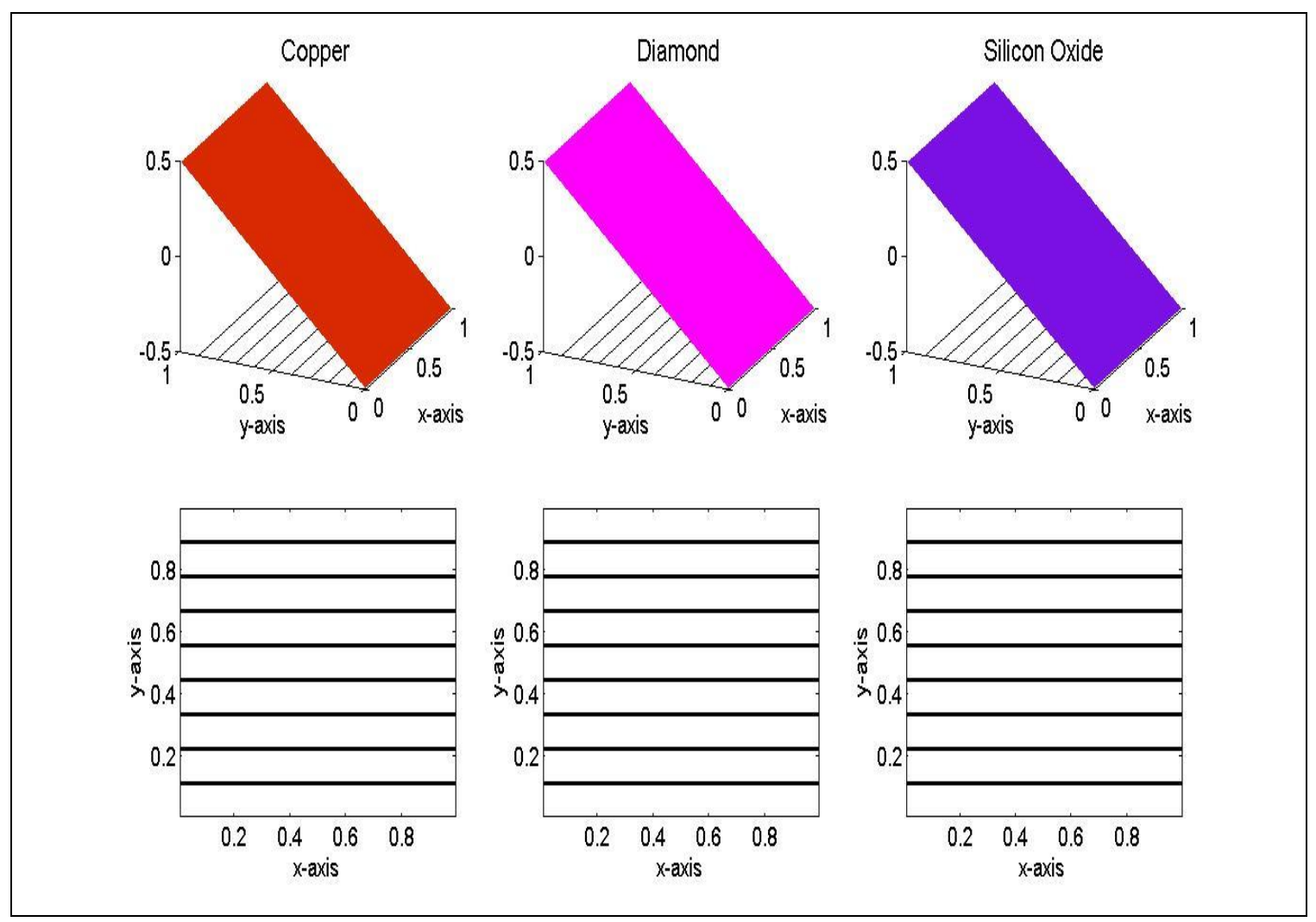

Figure 2a. Velocity, temperature and concentration contours using copper, diamond and silicon oxide nanoparticles.
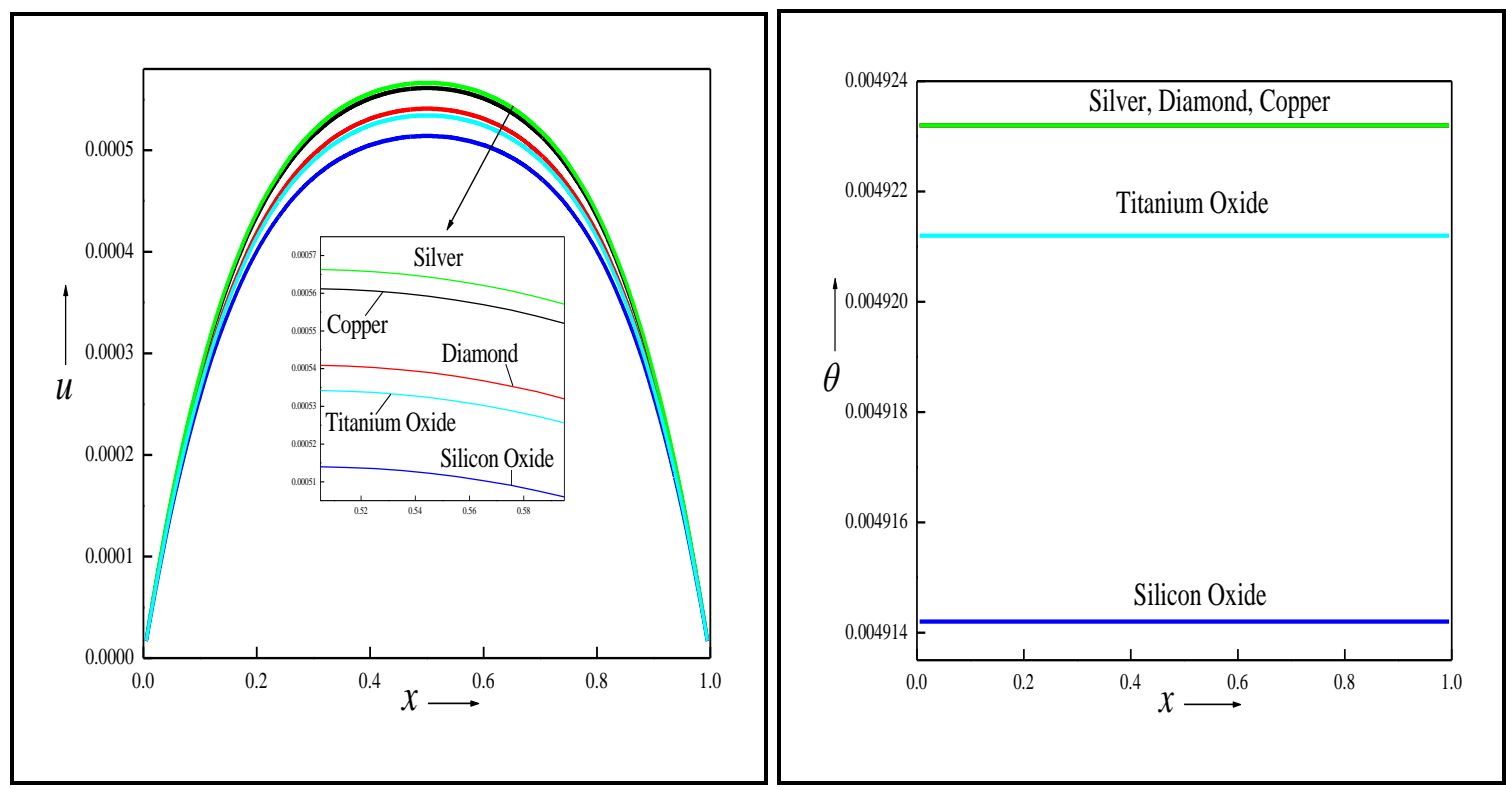


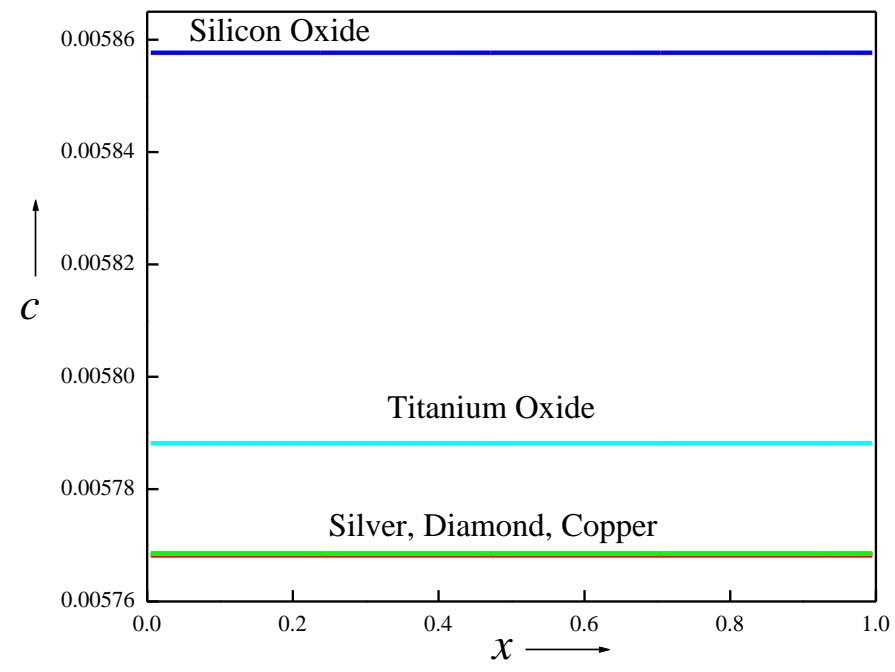

Figure $2 \mathbf{b}$. Velocity, temperature and concentration profiles for copper, silver, titanium oxide, diamond and silicon oxide nanoparticles.

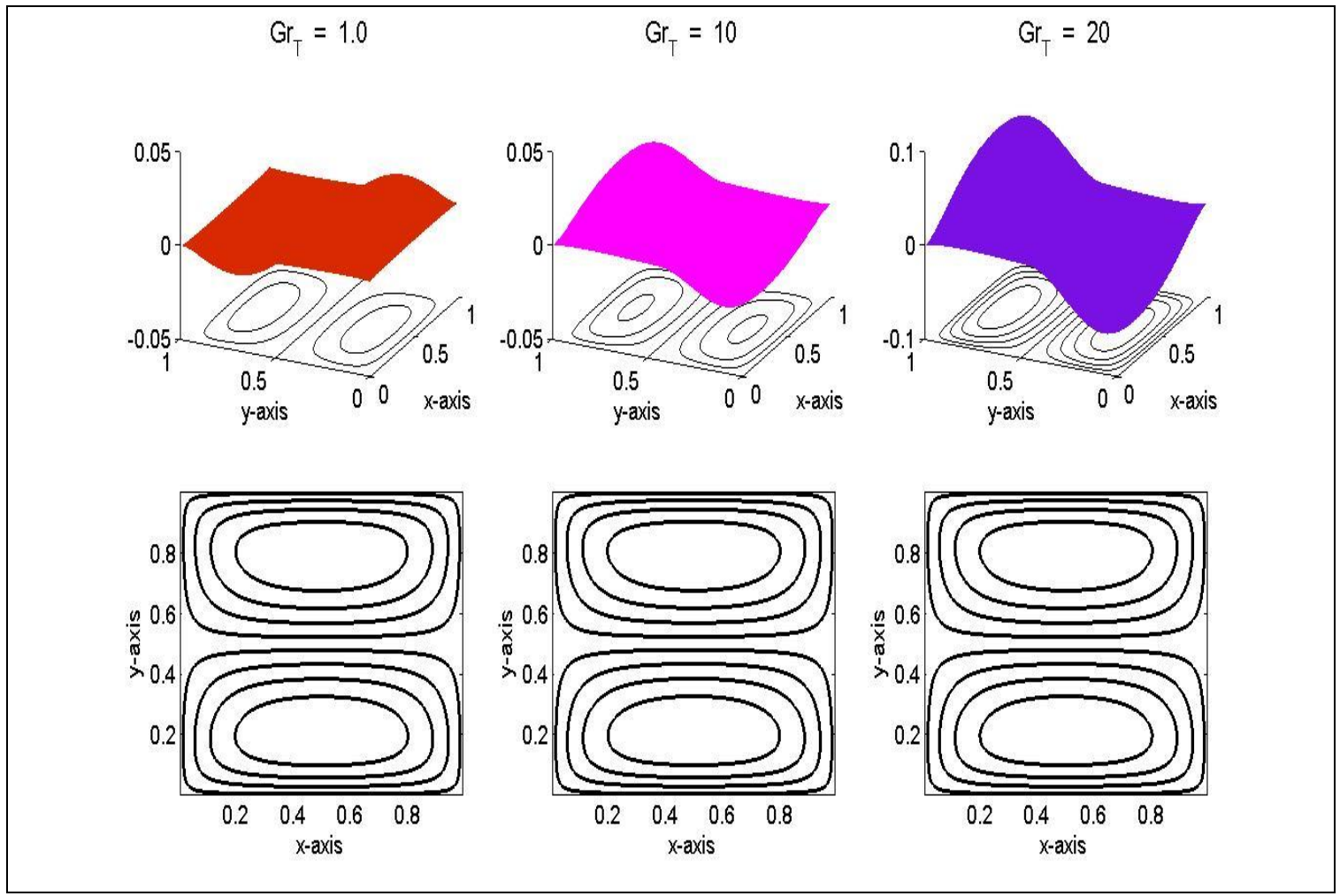




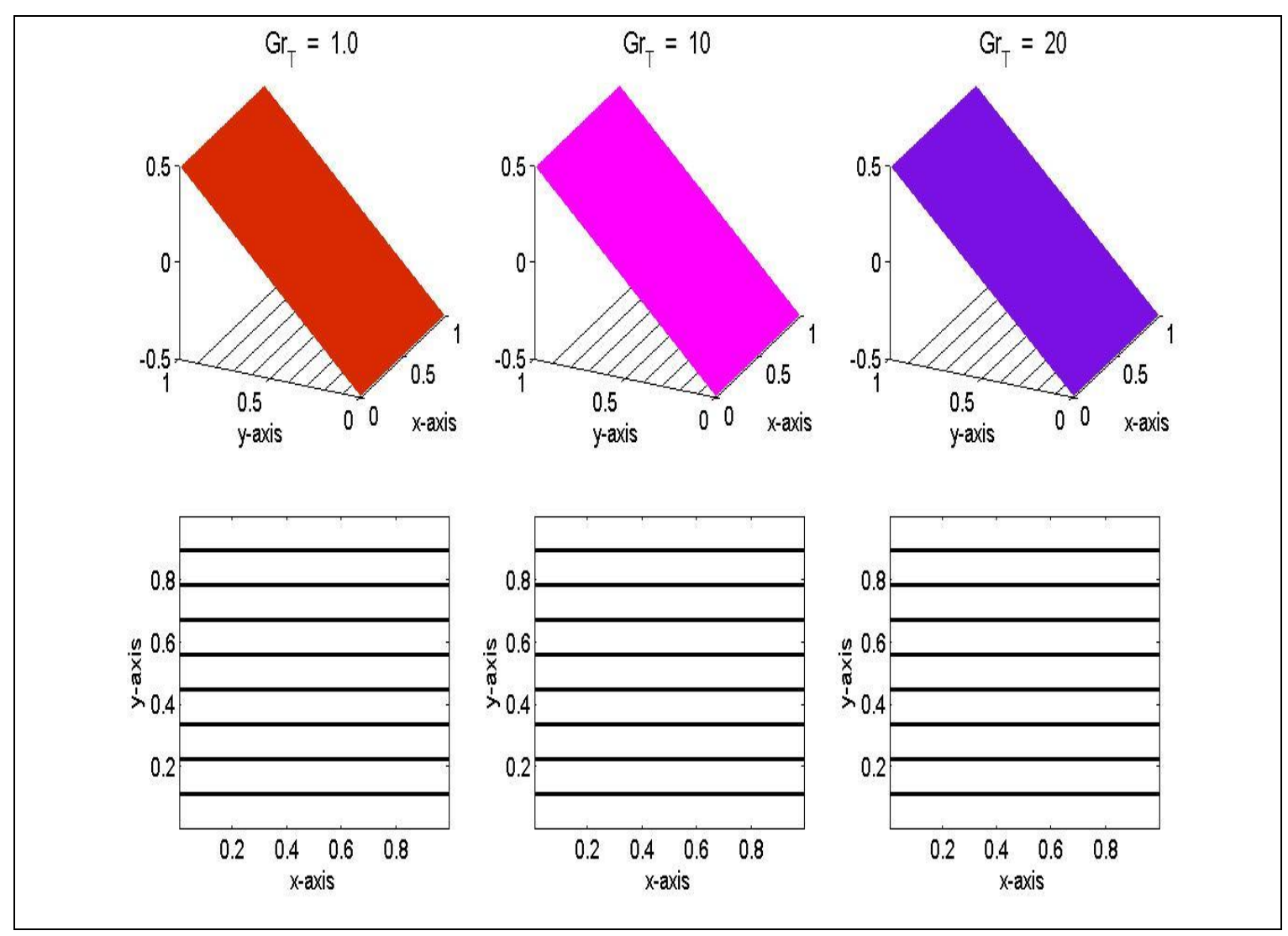

Figure 3a. Velocity and temperature contours for various values of $G r_{T}$.
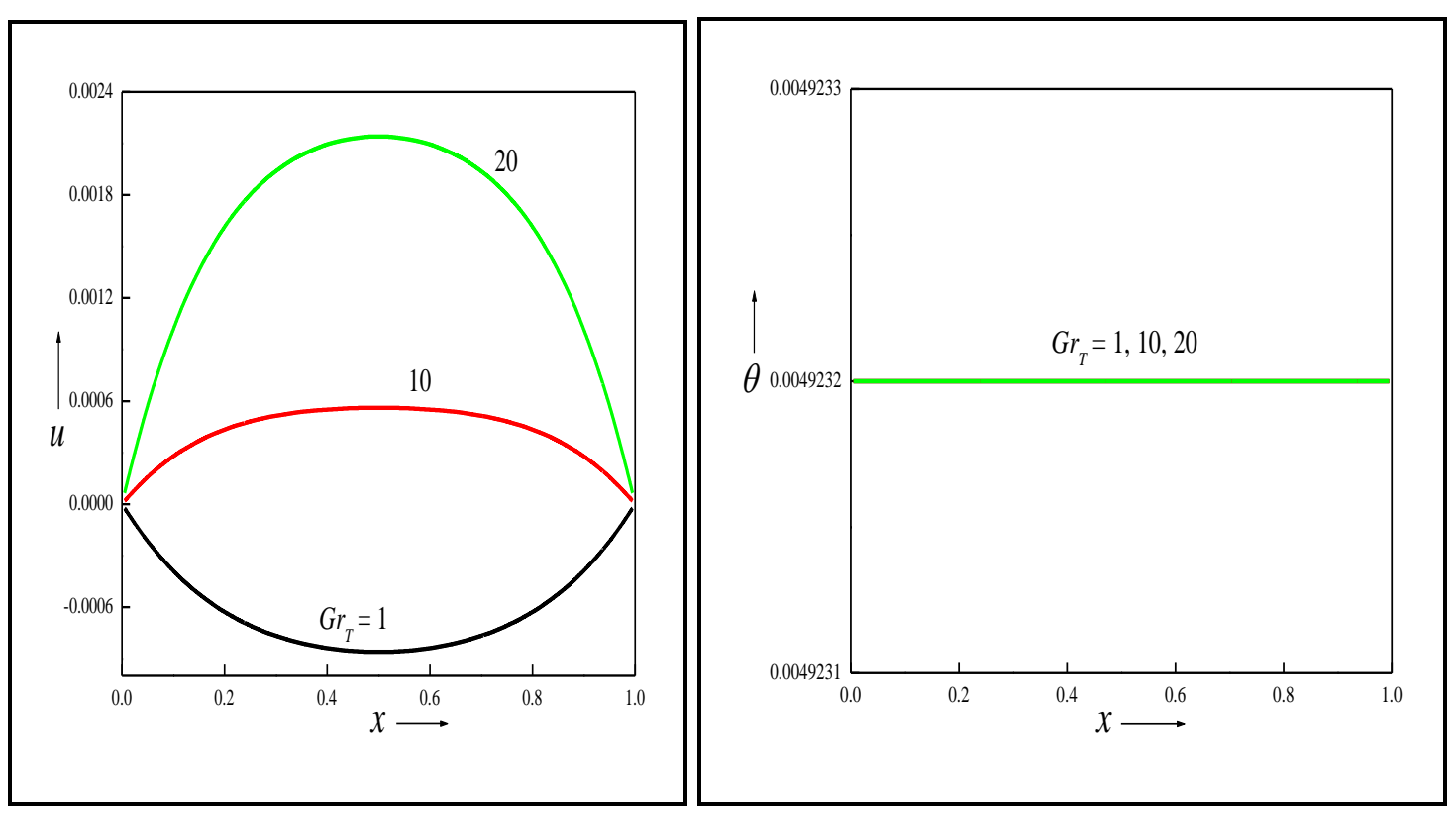


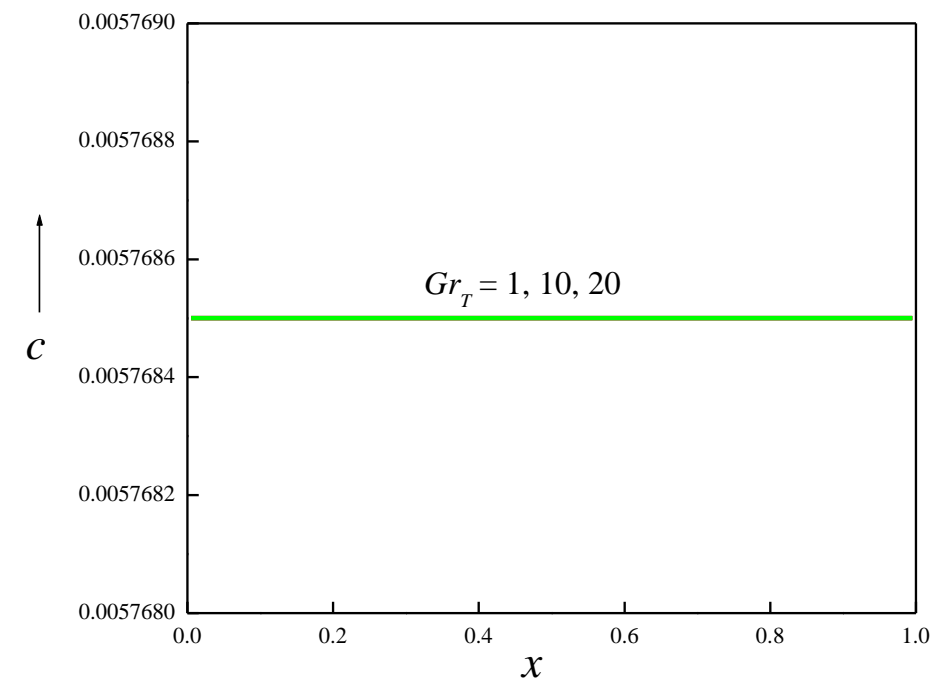

Figure 3b. Velocity, temperature and nanoparticle concentration sketches for various values of $G r_{T}$.

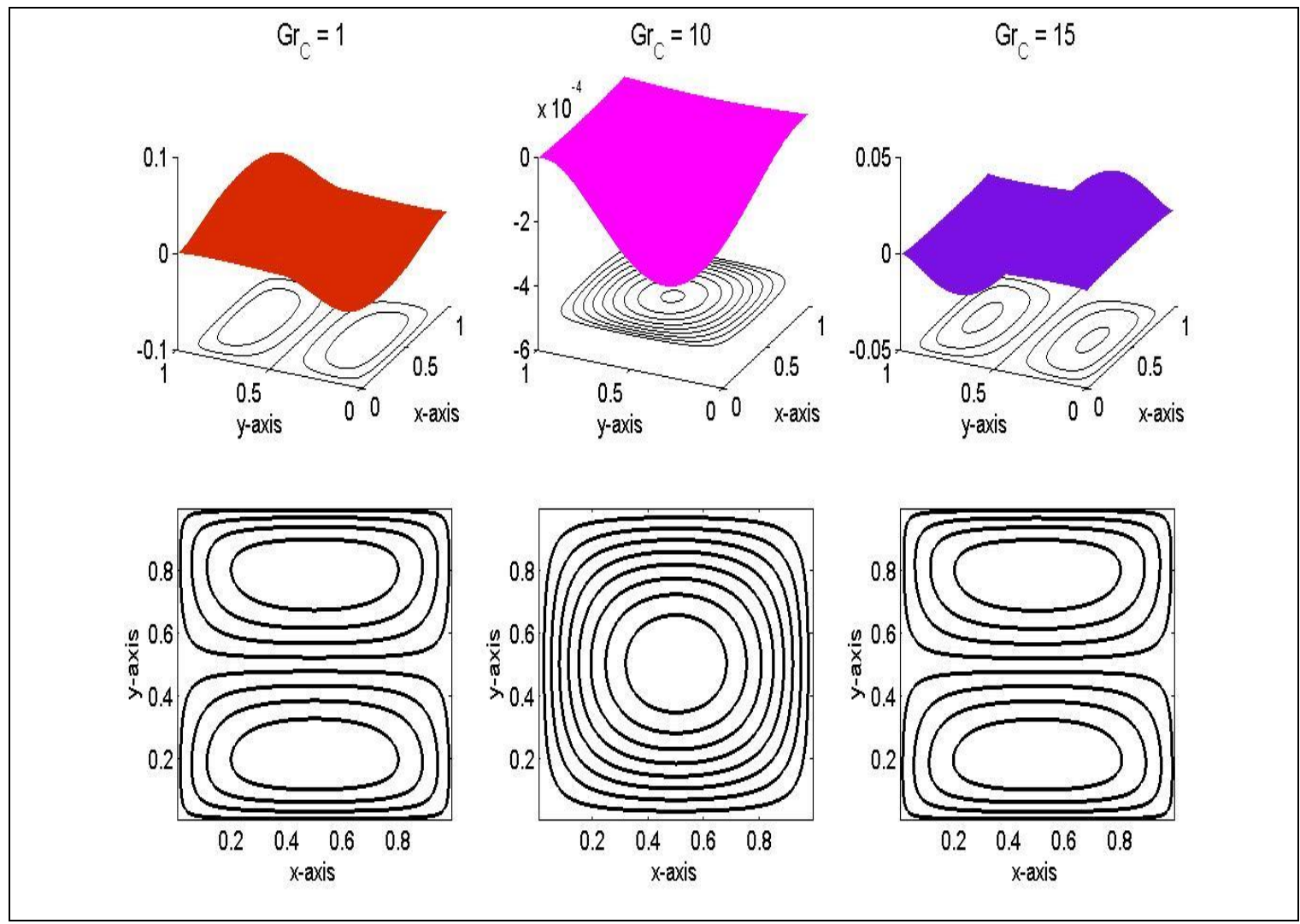

Figure 4a. Velocity contours for various values of $G r_{C}$ 


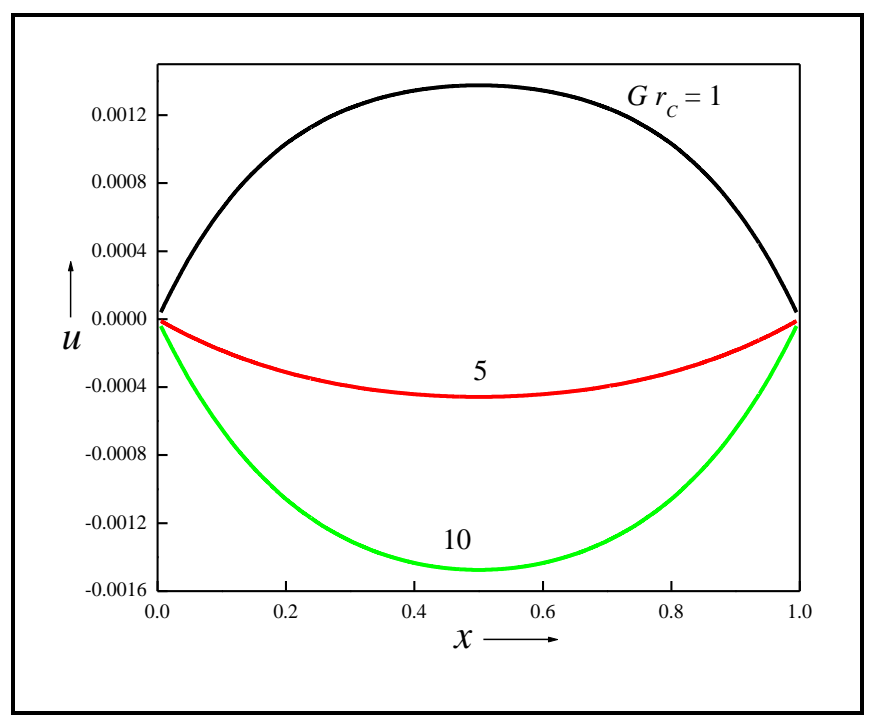

Figure $4 \mathbf{b}$. Velocity sketches for various values of $G r_{C}$

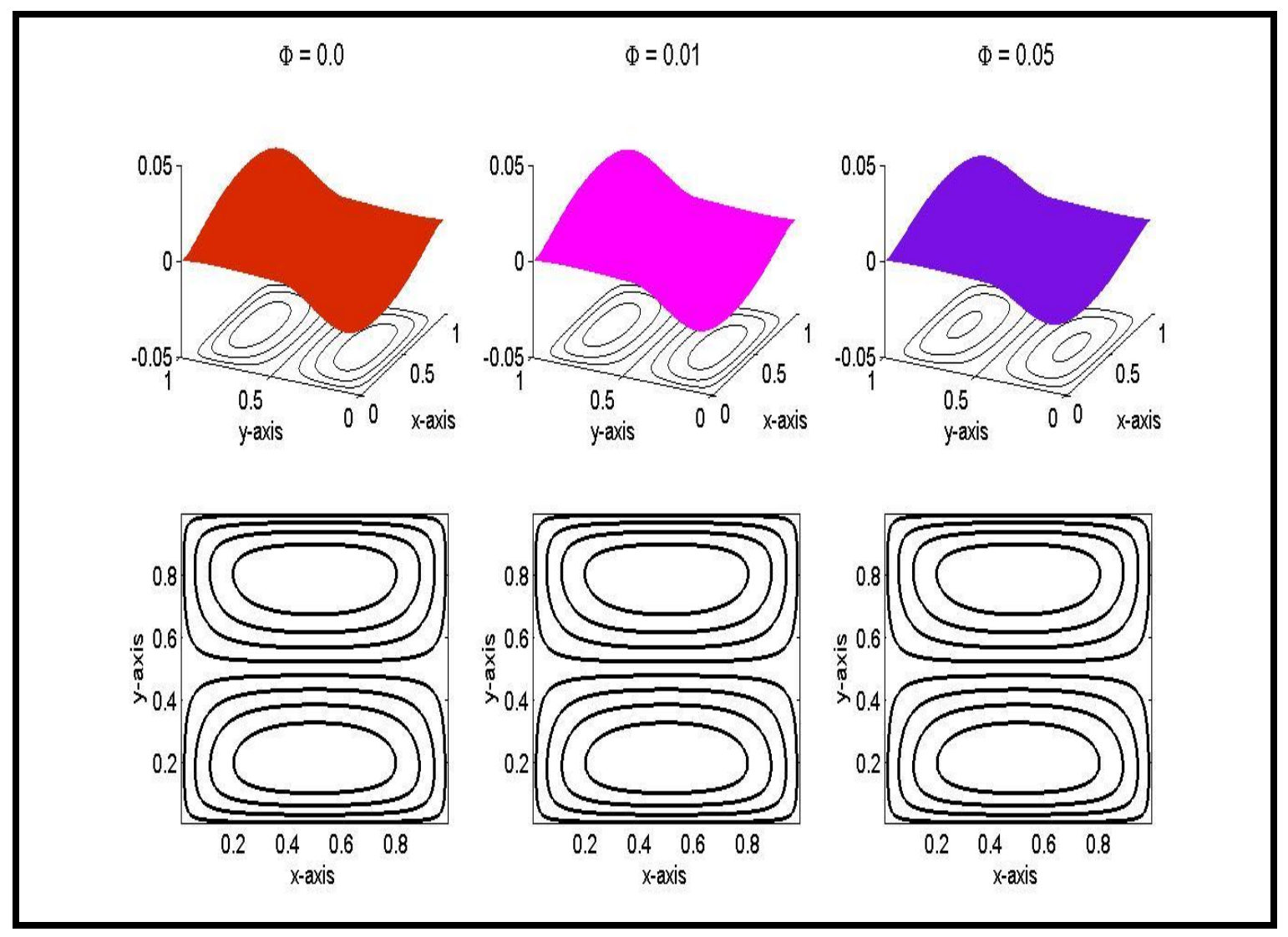

Figure 5a. Velocity and temperature contours for various values of $\phi$ 

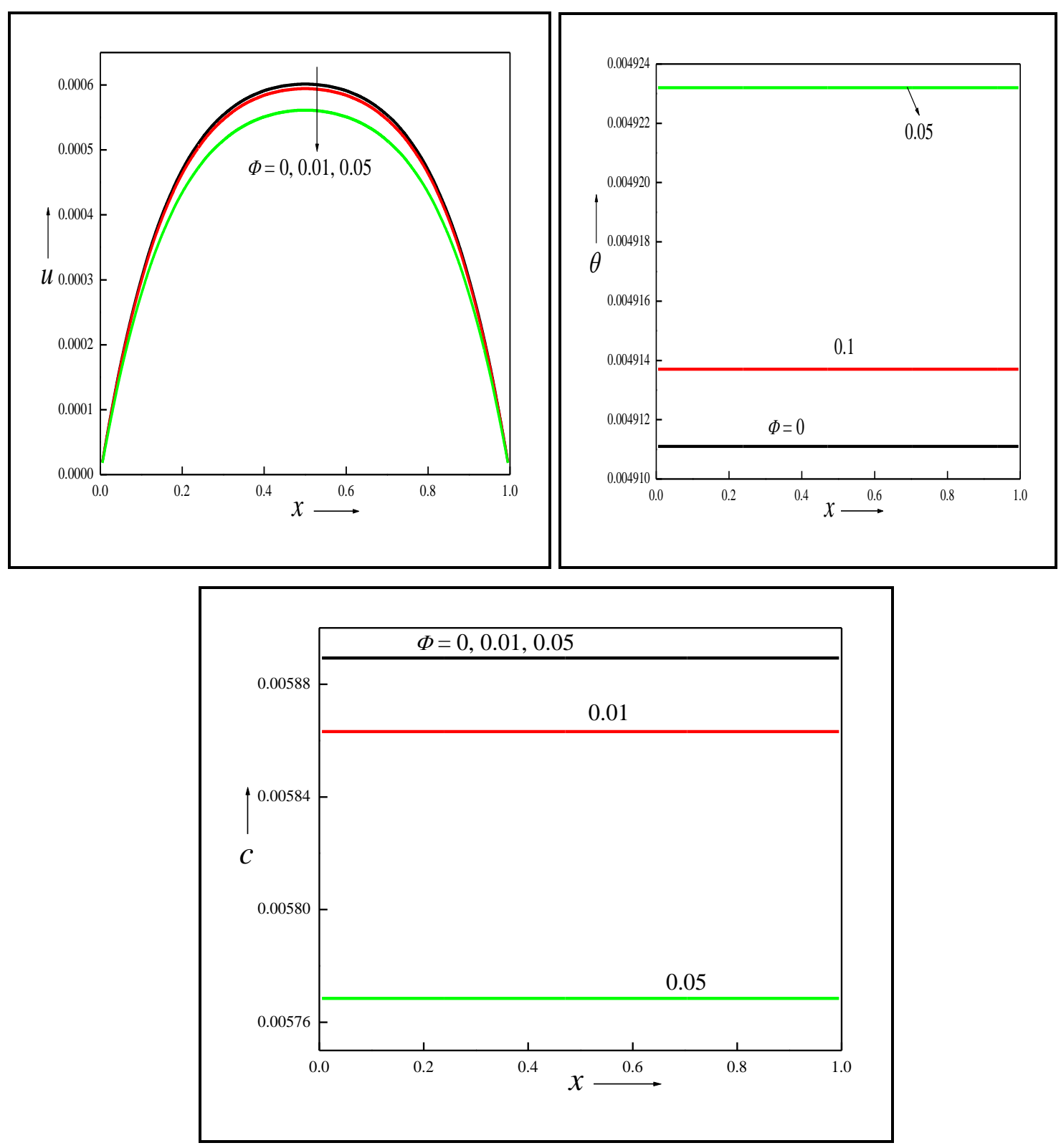

Figure 5b. Velocity, temperature and nanoparticle concentration sketches for various values of $\phi$ 


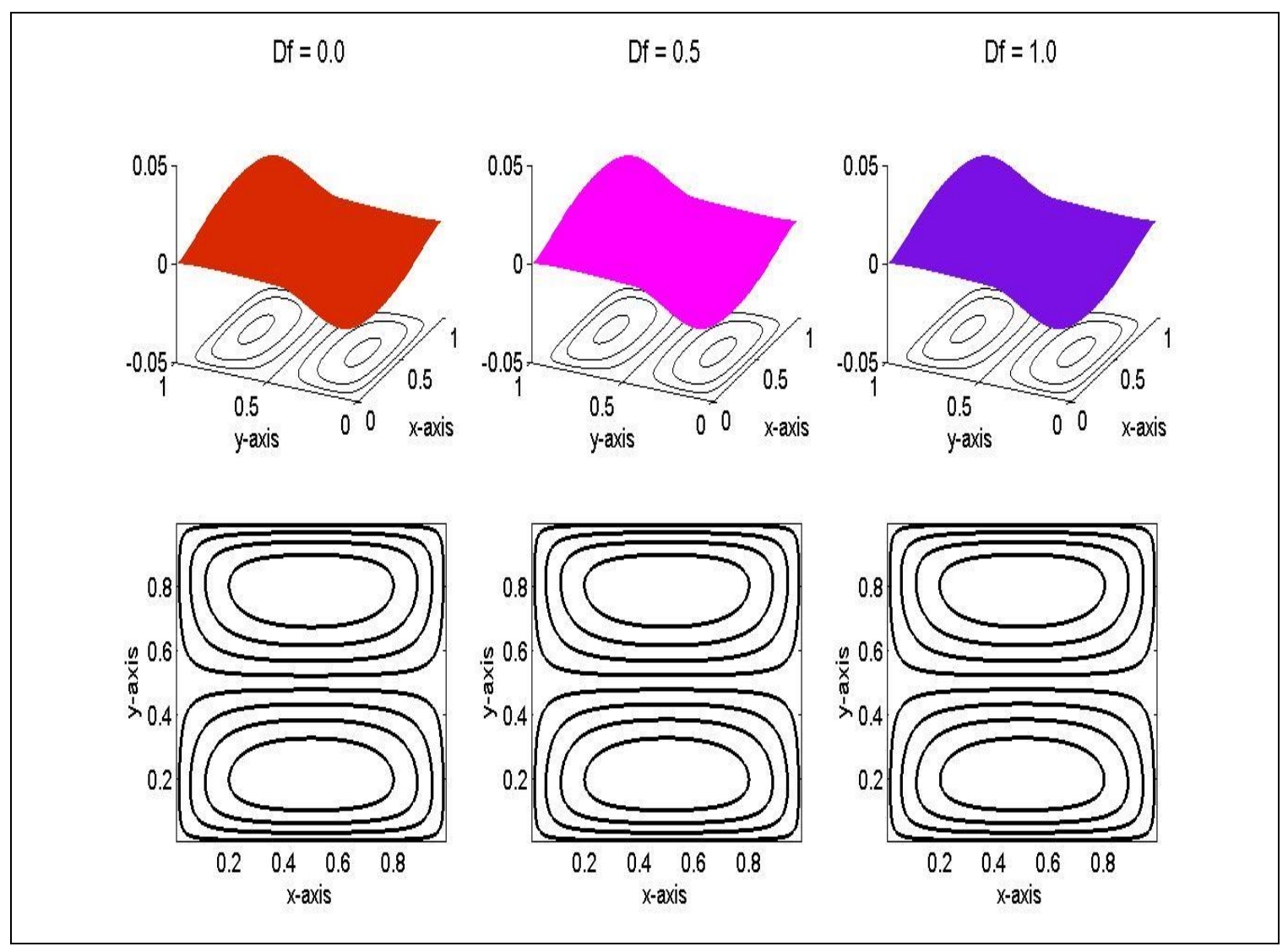

Figure 6a. Velocity contours for various values of $D f$
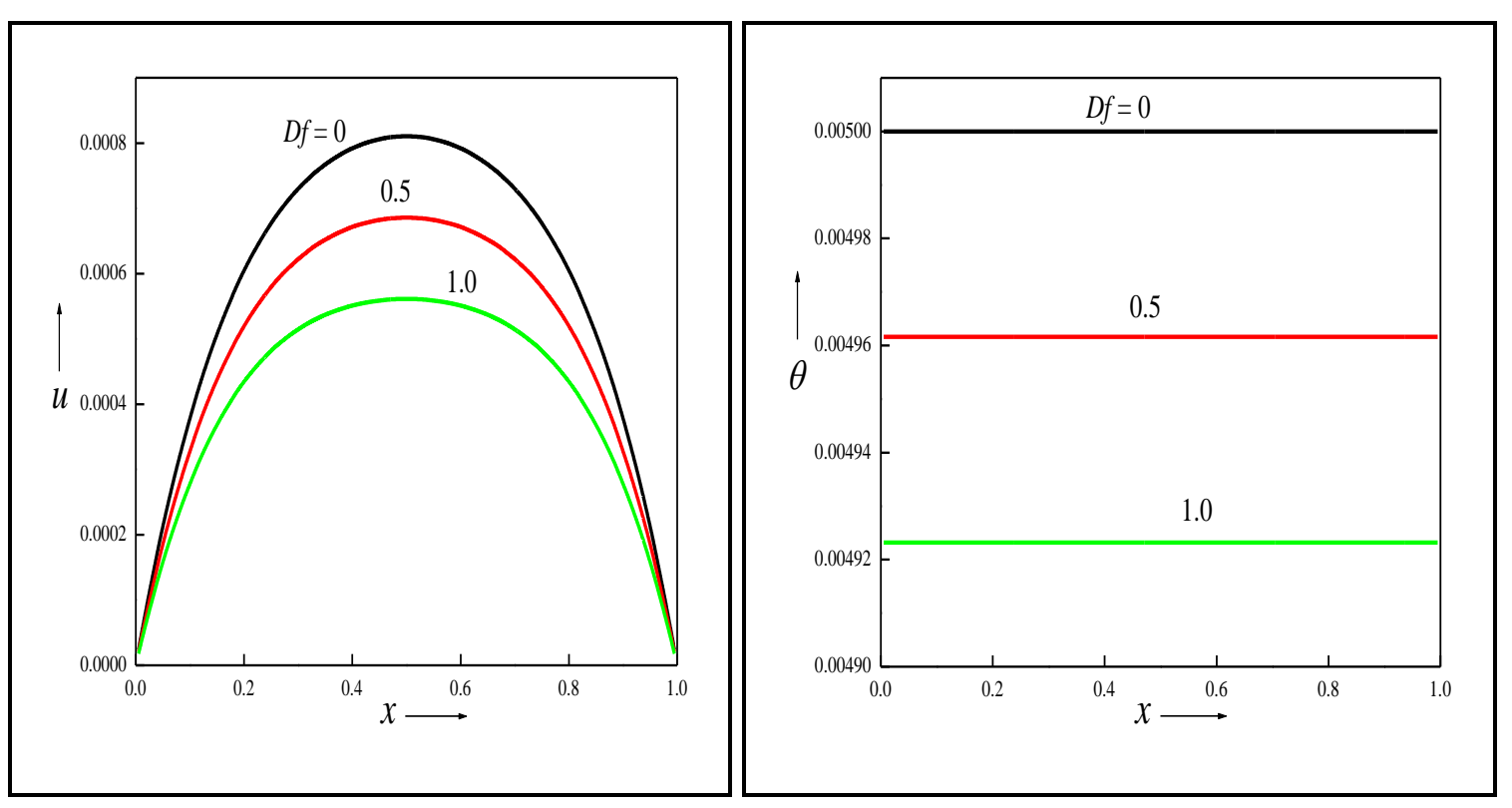


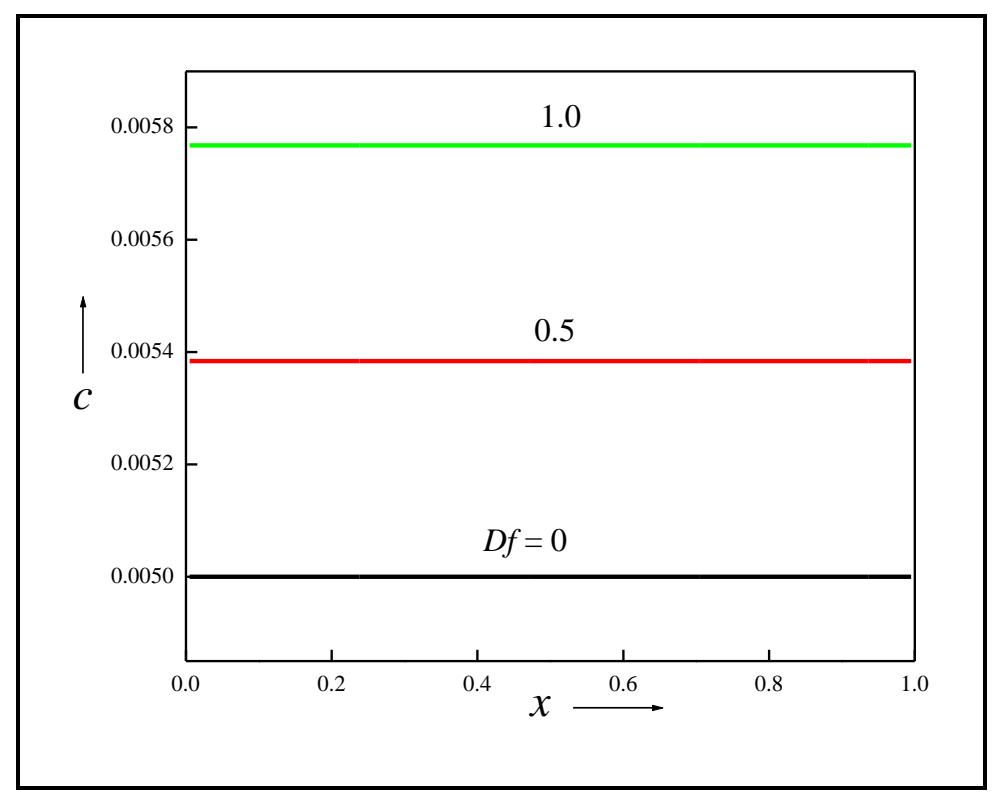

Figure 6b. Velocity, temperature and nanoparticle concentration distribution for various values of $D f$

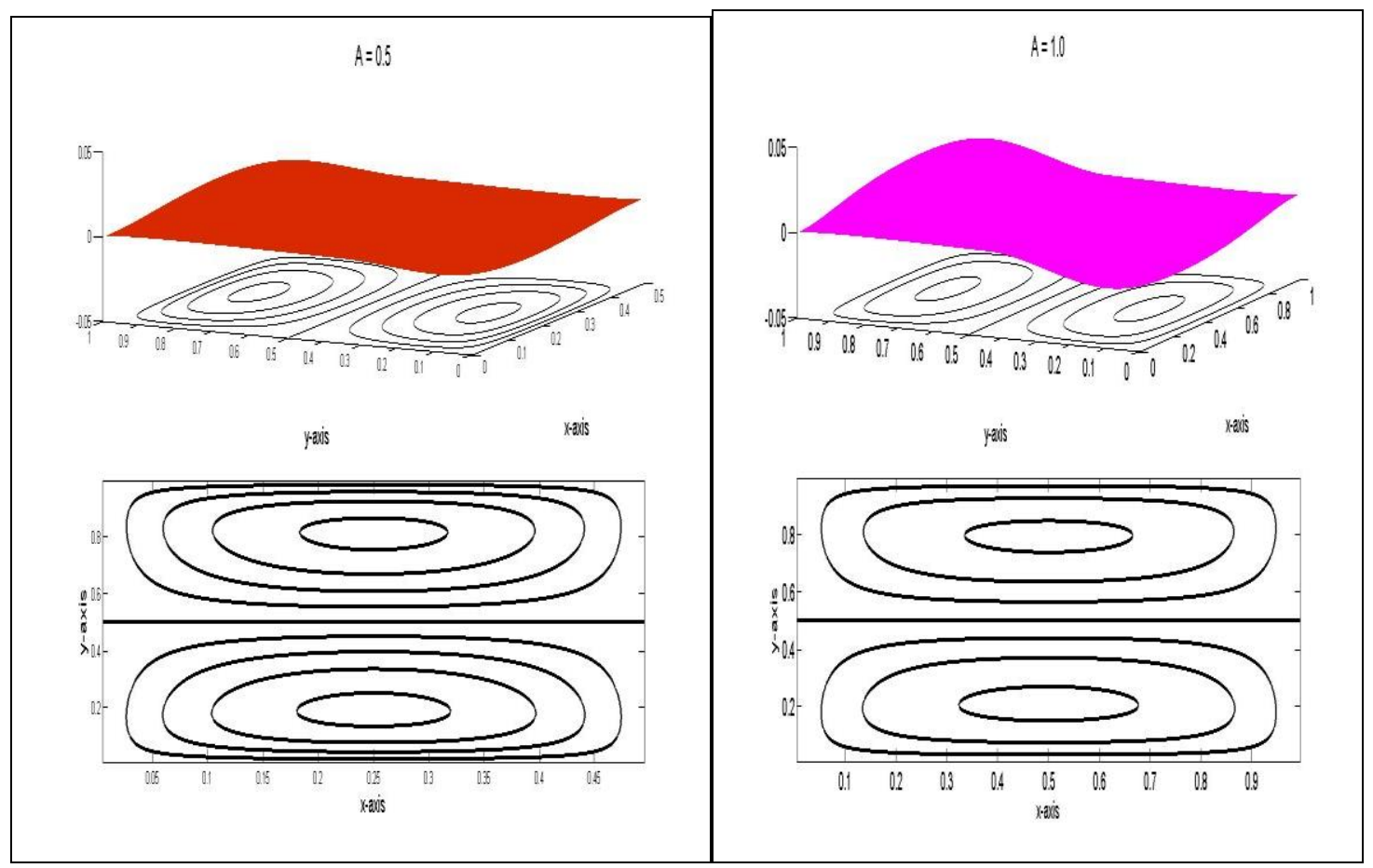




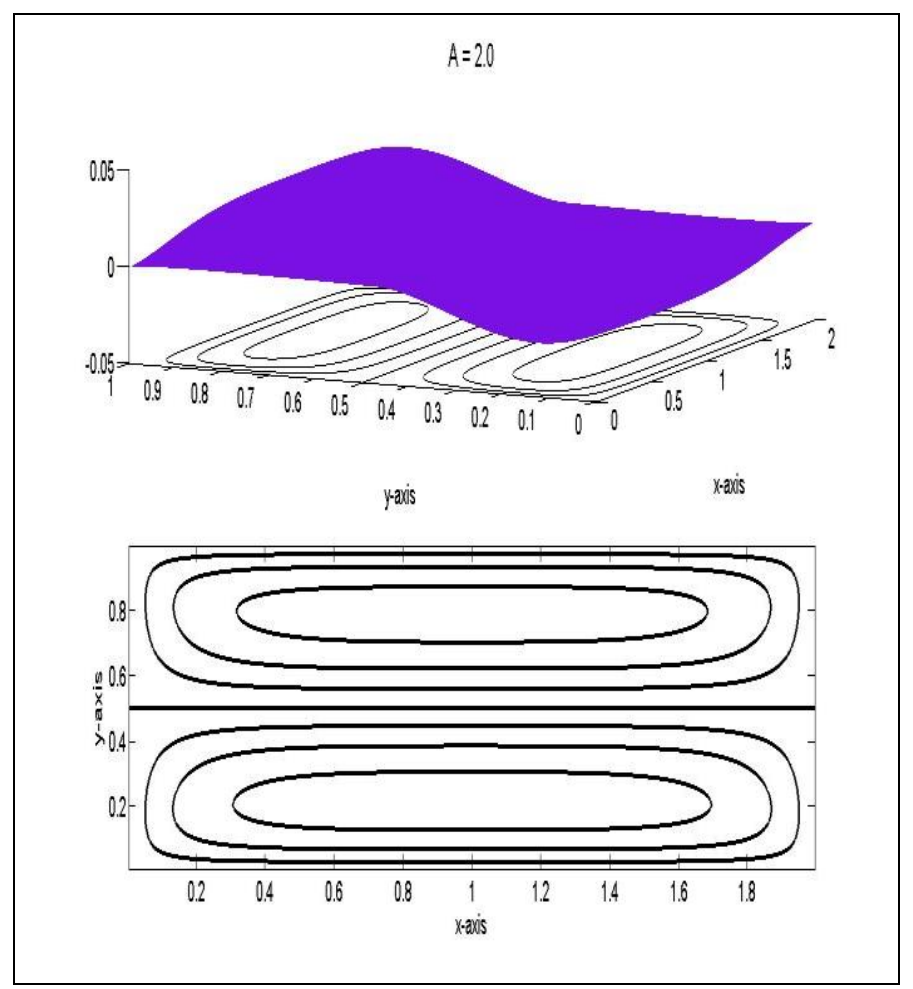

Figure 7a. Velocity contours for various values of $A$

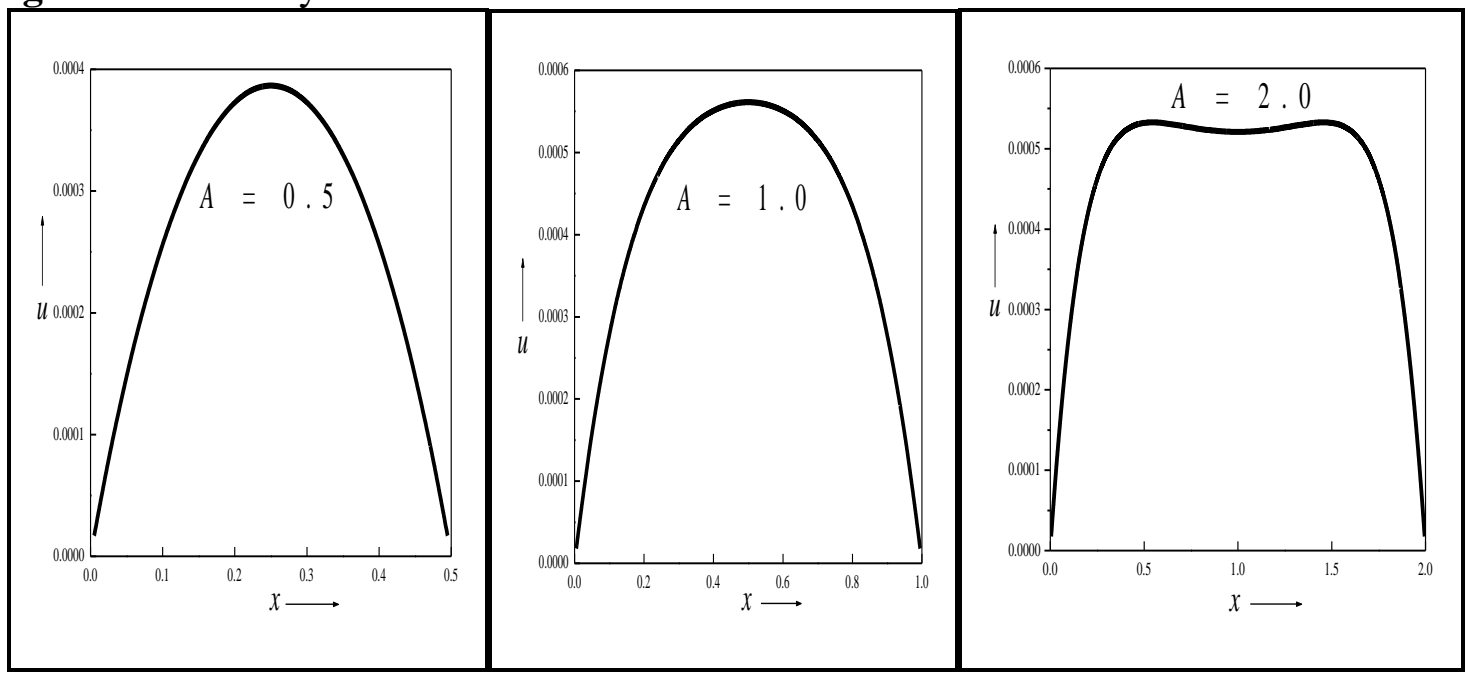




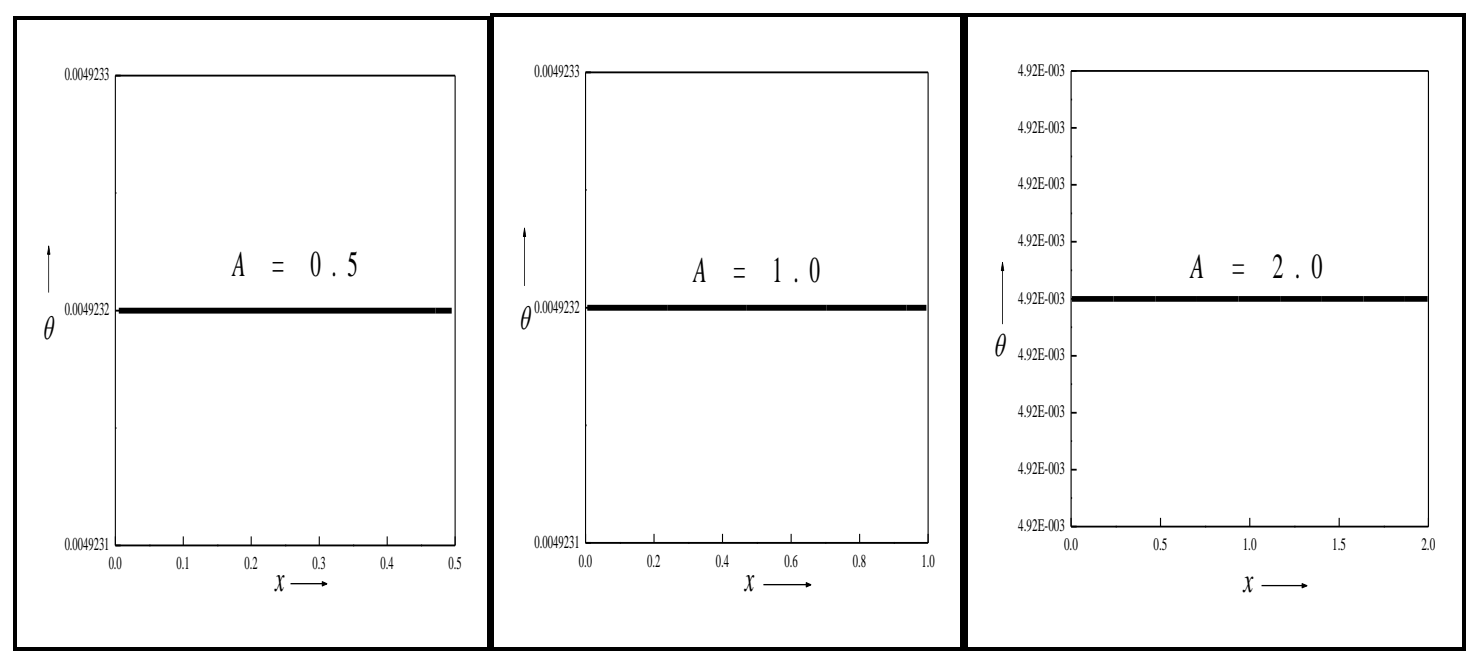

Figure 7b. Velocity and temperature profiles with $A$

Table 2. Volumetric flow rate and skin friction for copper-water nanofluid

\begin{tabular}{|c|c|c|c|c|c|}
\hline & $Q\left(10^{-4}\right)$ & $\left(\frac{d w}{d y}\right)_{y=0}$ & $\left(\frac{d w}{d y}\right)_{y=1}$ & $\left(\frac{d w}{d x}\right)_{x=0}\left(10^{-4}\right)$ & $\left(\frac{d w}{d x}\right)_{x=1}\left(10^{-4}\right)$ \\
\hline Nanoparticles & \multicolumn{5}{|l|}{} \\
\hline Copper & -1.1060 & -0.139152 & -0.138598 & -3.833612 & 3.833612 \\
\hline Diamond & -1.0653 & -0.134084 & -0.133551 & -3.692539 & 3.692539 \\
\hline Silver & -1.1160 & -0.140421 & -0.139862 & -3.868392 & 3.868392 \\
\hline $\mathrm{TiO}_{2}$ & -1.0923 & -0.133996 & -0.133449 & -3.786206 & 3.786206 \\
\hline $\mathrm{SiO}_{2}$ & -1.1920 & -0.134408 & -0.133811 & -4.132031 & 4.132031 \\
\hline $\mathrm{Gr}_{T}$ & \multicolumn{5}{|l|}{} \\
\hline 1 & -0.9401 & 0.110873 & 0.111344 & -3.258570 & 3.258570 \\
\hline 10 & -1.1060 & -0.139152 & -0.138598 & -3.833612 & 3.833612 \\
\hline 20 & -1.2903 & -0.416960 & -0.416313 & -4.472547 & 4.472547 \\
\hline$G r_{C}$ & \multicolumn{5}{|l|}{} \\
\hline 1 & -0.3686 & -0.250076 & -0.249891 & -1.277870 & 1.277870 \\
\hline 10 & -2.0276 & -0.000498 & 0.000517 & -7.028288 & 7.028288 \\
\hline 15 & -2.9493 & 0.138156 & 0.139633 & -10.222963 & 10.222969 \\
\hline$\phi$ & \multicolumn{5}{|l|}{} \\
\hline 0 & -1.4743 & -0.160358 & -0.159620 & -5.110504 & 5.110504 \\
\hline 0.01 & -1.3920 & -0.155956 & -0.155259 & -4.824982 & 4.824982 \\
\hline 0.05 & -1.1060 & -0.139152 & -0.138598 & -3.833612 & 3.833612 \\
\hline$D f$ & \multicolumn{5}{|l|}{} \\
\hline 0 & 0.0000 & -0.138880 & -0.138880 & -1.395133 & 1.361632 \\
\hline 0.5 & -0.5552 & -0.139016 & -0.138739 & -1.916732 & 1.916732 \\
\hline 1.0 & -1.1060 & -0.139152 & -0.138598 & -3.833612 & 3.833612 \\
\hline
\end{tabular}




\begin{tabular}{|c|c|c|c|c|c|}
\hline$P r$ & \multicolumn{5}{|l|}{} \\
\hline 0 & -0.2925 & -0.138952 & -0.138806 & -1.014126 & 1.014126 \\
\hline 1.0 & -0.8777 & -0.139096 & -0.138657 & -3.042501 & 3.04250 \\
\hline 7.0 & -1.4630 & -0.139240 & -0.138507 & -5.071039 & 5.071039 \\
\hline$S r$ & \multicolumn{5}{|c|}{} \\
\hline 0 & -0.1843 & -0.138926 & -0.138833 & -0.638886 & 0.638886 \\
\hline 1 & -0.6451 & -0.139039 & -0.138716 & -2.236188 & 2.236188 \\
\hline 5 & -2.4887 & -0.139492 & -0.138246 & -8.626598 & 8.626598 \\
\hline$S c$ & \multicolumn{5}{|c|}{} \\
\hline 0 & -0.1843 & -0.138926 & -0.138833 & -0.638886 & 0.638886 \\
\hline 1 & -0.6451 & -0.139039 & -0.138716 & -2.236188 & 2.236188 \\
\hline 5 & -2.4887 & -0.139492 & -0.138246 & -8.626598 & 8.626598 \\
\hline$A$ & \multicolumn{5}{|c|}{} \\
\hline 0.5 & -4.3964 & -0.102624 & -0.102402 & -2.744777 & 2.744777 \\
\hline 1.0 & -1.1060 & -0.139152 & -0.138598 & -3.833612 & 3.833612 \\
\hline 2.0 & -1.8136 & -0.160231 & -0.159327 & -4.163215 & 4.163215 \\
\hline
\end{tabular}

Table 3. Nusselt number for water-copper nanofluid

\begin{tabular}{|c|r|r|}
\hline & $\left.\frac{d \theta}{d y}\right|_{y=0}$ & $\left.\frac{d \theta}{d y}\right|_{y=1}$ \\
\hline Nanoparticles & \multicolumn{2}{|l|}{} \\
\hline Copper & 0.578746005975844 & 0.578746006039670 \\
\hline Diamond & 0.578974186845769 & 0.578974186909538 \\
\hline Silver & 0.578771668876074 & 0.578771668939665 \\
\hline $\mathrm{TiO}_{2}$ & 0.564255580401620 & 0.564255580463591 \\
\hline $\mathrm{SiO}_{2}$ & 0.518548813488192 & 0.518548813544569 \\
\hline$G r_{T}$ & \multicolumn{2}{|l}{} \\
\hline 1 & 0.578746005975844 & 0.578746006039670 \\
\hline 10 & 0.578746005975844 & 0.578746006039670 \\
\hline 20 & 0.578746005983829 & 0.578746006031875 \\
\hline$G r_{C}$ & \multicolumn{2}{|l}{} \\
\hline 1 & 0.578746005975844 & 0.578746006039670 \\
\hline 10 & 0.578746005975844 & 0.578746006039670 \\
\hline 15 & 0.578746005975844 & 0.578746006039670 \\
\hline$\phi$ & \multicolumn{2}{|l}{} \\
\hline 0 & 0.500179413113745 & 0.500179413167717 \\
\hline 0.01 & 0.515260789410006 & 0.515260789465842 \\
\hline 0.05 & 0.578746005975844 & 0.578746006039670 \\
\hline$D f$ & \multicolumn{2}{|l}{} \\
\hline 0 & 0.578566565229622 & 0.578566565302447 \\
\hline
\end{tabular}




\begin{tabular}{|c|c|c|}
\hline 0.5 & 0.578656329512178 & 0.578656329580232 \\
\hline 1.0 & 0.578746005975844 & 0.578746006039670 \\
\hline$P r$ & \multicolumn{2}{|c|}{} \\
\hline 0 & 0.578614070498587 & 0.578614070568742 \\
\hline 1.0 & 0.578709007286562 & 0.578709007351978 \\
\hline 7.0 & 0.578803845724925 & 0.578803845786173 \\
\hline$S r$ & $|2|$ \\
\hline 0 & 0.578746181598002 & 0.578746181669840 \\
\hline 1 & 0.578746093792582 & 0.578746093860193 \\
\hline 5 & 0.578745742459675 & 0.578745742513047 \\
\hline$S c$ & \multicolumn{2}{|l|}{} \\
\hline 0 & 0.578746181598002 & 0.578746181669840 \\
\hline 1 & 0.578746093792582 & 0.578746093860193 \\
\hline 5 & 0.578745742459675 & 0.578745742513047 \\
\hline$A$ & 0.578746005975433 & 0.578746006040076 \\
\hline 0.5 & 0.578746005975844 & 0.578746006039670 \\
\hline 1.0 & 0.578746005987664 & 0.578746006028130 \\
\hline 2.0 &
\end{tabular}

Table 4. Sherwood number (wall mass transfer gradient) for water-copper nanofluid

\begin{tabular}{|c|c|c|}
\hline & $\left.\frac{d c}{d y}\right|_{y=0}$ & $\left.\frac{d c}{d y}\right|_{y=1}$ \\
\hline \multicolumn{3}{|l|}{ Nanoparticles } \\
\hline Copper & 0.498449264474509 & 0.498449264537100 \\
\hline Diamond & 0.498449875229360 & 0.498449875292323 \\
\hline Silver & 0.498449333188210 & 0.498449333251021 \\
\hline $\mathrm{TiO}_{2}$ & 0.498409467986961 & 0.498409468049760 \\
\hline $\mathrm{SiO}_{2}$ & 0.498269377500321 & 0.498269377562944 \\
\hline \multicolumn{3}{|l|}{$G r_{T}$} \\
\hline 1 & 0.498449264474509 & 0.498449264537100 \\
\hline 10 & 0.498449264474509 & 0.498449264537100 \\
\hline 20 & 0.498449264482394 & 0.498449264529428 \\
\hline \multicolumn{3}{|l|}{$G r_{C}$} \\
\hline 1 & 0.498449264474509 & 0.498449264537100 \\
\hline 10 & 0.498449264474509 & 0.498449264537100 \\
\hline 15 & 0.498449264474509 & 0.498449264537100 \\
\hline \multicolumn{3}{|c|}{ 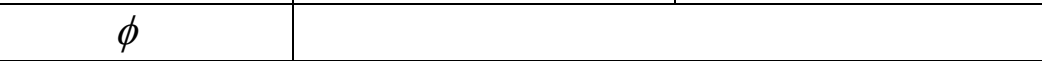 } \\
\hline 0 & 0.498205868558296 & 0.498205868621438 \\
\hline 0.01 & 0.498258342217574 & 0.498258342279895 \\
\hline 0.05 & 0.498449264474509 & 0.498449264537100 \\
\hline \multicolumn{3}{|l|}{$D f$} \\
\hline 0 & 0.499999999968229 & 0.500000000031163 \\
\hline
\end{tabular}




\begin{tabular}{|c|c|c|}
\hline 0.5 & 0.499224252755222 & 0.499224252817583 \\
\hline 1.0 & 0.498449264474509 & 0.498449264537100 \\
\hline$P r$ & $0 . \mid$ \\
\hline 2 & 0.499589457167556 & 0.499589457230535 \\
\hline 6 & 0.498769008914318 & 0.498769008977202 \\
\hline 10 & 0.497949410600413 & 0.497949410663649 \\
\hline$S r$ & $\mid$ \\
\hline 0 & 0.499999999968226 & 0.500000000031163 \\
\hline 1 & 0.499224252754838 & 0.499224252817656 \\
\hline 5 & 0.496128854647523 & 0.496128854707979 \\
\hline$S c$ & 0.499999999968226 & 0.500000000031163 \\
\hline 0 & 0.499224252754838 & 0.499224252817656 \\
\hline 1 & 0.496128854647523 & 0.496128854707979 \\
\hline 5 & 0.498449264474080 & 0.498449264537522 \\
\hline$A$ & 0.498449264474509 & 0.498449264537100 \\
\hline 0.5 & 0.498449264486193 & 0.498449264525723 \\
\hline 1.0
\end{tabular}

Figures 2a display the velocity, temperature and concentration contours using copper, diamond and $\mathrm{SiO}_{2}$ as nanoparticles with aqueous base fluid. The contours are not strongly modified by using different nanoparticles. The flow parameters are anti symmetric. It can also be observed that there is a symmetric distribution at the midsection of the duct $(y=0.5)$. The intensity of the contours is reduced (i.e. they are relaxed for carbon-based nanoparticles) for $\mathrm{SiO}_{2}$ and diamond nanoparticles, relative to copper nanoparticle. The thermal and concentration contours are identical for copper, diamond and $\mathrm{SiO}_{2}$ nanoparticles and decrease linearly from the right duct wall $(y=1)$ to the left duct wall $(y=0)$. Figure $\mathbf{2 b}$ is mapped to examine the impact of copper, diamond, $\mathrm{SiO}_{2}, \mathrm{TiO}_{2}$ and silver nanoparticles keeping $y$ fixed and varying $x$. Onedimensional graphs clearly indicate that the magnitude of velocity decreases sequentially with silver, copper, diamond, $\mathrm{TiO}_{2}$ and $\mathrm{SiO}_{2}$ respectively i.e. these nanoparticles produce progressively greater deceleration. The silver, diamond and copper shows that the optimum values are obtained for temperature and are minimal for $\mathrm{TiO}_{2}$ and $\mathrm{SiO}_{2}$, respectively. The nanoparticle concentration profiles decrease, sequentially for $\mathrm{SiO}_{2}$, $\mathrm{TiO}_{2}$, silver, diamond and copper respectively. 
The flow contours for various values of thermal Grashof number $G r_{T}$ for water as the base fluid with copper nanoparticles are shown in Figs. 3a, b. The response of thermal Grashof number $G r_{T}$ is to boost the velocity in both upward and downward directions. For the effects of thermal Grashof number $G r_{T}$ the thermal distributions are analogous and exhibit linear distributions for all values of $G r_{T}$. The concentration contours for various thermal Grashof number $G r_{T}$ show similar nature as that of temperature contours and are therefore not included. The effect of thermal Grashof number $G r_{T}$ can be examined clearly in one-dimensional graphs as shown in Fig. $3 \mathrm{~b}$. Increasing the thermal Grashof number induces strong flow acceleration owing to the fact that $G r_{T}>0$ corresponds to buoyancy-assisted flow and hence velocity increases. The effect of $G r_{T}$ on temperature and nanoparticle concentration profiles are invariant and this is largely attributable to the fact that the viscous dissipation is neglected in the energy equation.

Fig. 4a, $b$ is plotted to show the velocity contours and profiles, respectively, for the effect of nano-species concentration Grashof number $G r_{C}$. It is evident that the velocity contours are symmetric in both upward and downward directions for $G r_{C}=1$ (viscous and species buoyancy forces are equivalent) whereas for $G r_{C}=10$ the intensity of velocity is more in the sinking direction and for $G r_{C}=15$ the magnitude of velocity contours decreases substantially in both skyward and sinking directions. Inspection of the one-dimensional graphs - keeping $y$ fixed and varying $x$, clearly visualize that as $G r_{C}$ increases the velocity decreases. The temperature and concentration contours and profiles are not displayed graphically as they exhibit similar topologies to the temperature and concentration contours as that on $G r_{T}$.

The impact of $\phi$ (solid volume fraction) is plotted in Figs. 5a, b. The velocity contours resemble one another for various values of $\phi$. The temperature and concentration contours obtained are similar to $G r_{T}$ and therefore not presented. However, the effect of solid volume fraction can be clearly identified in the onedimensional graphs which demonstrate that the velocity and concentration profiles are 
suppressed whereas the temperature field is enhanced with increasing solid volume fraction $\phi$. This is physically attributable to the fact that adding nanoparticles to a viscous fluid will result in suspensions (similar to the effects of non-Newtonian fluid); the additives will result in reduction of velocity since viscous shear rate and momentum diffusion are altered.

The effect of Dufour parameter $D f$ on the flow is presented in Figs. 6a, b. The flow contours are symmetric in both upward and downward directions with increment in Dufour coefficient $D f$. The temperature and concentration contours are similar to the contours on $G r_{T}$ and hence not shown. Figure $6 \mathrm{~b}$ indicates that the velocity and temperature profiles are depleted whereas the concentration profiles are enhanced with stronger diffuso-thermal effect i. e. larger $D f$. The effects of $S c$ and $S r$ are similar to those computed for $D f$ and hence are not shown in graphical form.

The effect of aspect ratio $A$ on the transport characteristics is illustrated in Figs. 7a, b. The velocity contours assume plateau topologies i. e. flat distributions, for large values of $A$ when compared with small values of $A$. The temperature and concentration contours are not markedly influenced by the geometry of the duct (similar to $G r_{T}$ ). The one-dimensional graph for velocity also indicates that the velocity distribution is constricted for smaller values of $A$ and is naturally expanded across the duct width as $A$ increases, with concomitant narrowing and relaxation in the contours. The temperature profiles are not varied by changing the width of the duct.

Tables 2,3 and 4 document the impact of physical properties of the base fluid and different types nanoparticles on the volumetric flow rate, skin friction, Nusselt number and Sherwood number. Further the effect of thermal Grashof number $G r_{T}$, concentration Grashof number $G r_{C}$, solid volume fraction $\phi$, Dufour parameter $D f$, Prandtl number $\operatorname{Pr}$, Soret parameter $S r$, Schmidt parameter $S c$ and aspect ratio $A$ on the volumetric flow rate, skin friction, Nusselt number and Sherwood number are also computed in these Tables with water as the base fluid and copper nanoparticles.

Table 2 clearly indicates that diamond nanoparticles have the lowest volumetric flow rate and $\mathrm{SiO}_{2}$ nanoparticles achieve the highest volumetric flow rate when 
compared with copper, silver and $\mathrm{TiO}_{2}$ nanoparticles. Further it is observed that the volumetric flow rate increases with $G r_{T}, G r_{C}, D f, \operatorname{Pr}, S r, S c, A$ whereas it is depressed with an increment in $\phi$. The skin friction $\frac{\partial w}{\partial y}$ at $y=0$ achieves an optimal value for silver and minimum value for $\mathrm{TiO}_{2}$; furthermore, skin friction increases in magnitude (flow acceleration at the left duct wall) with $G r_{T}, G r_{C}, D f, \operatorname{Pr}, S r, S c, A$ and decreases (flow retardation at the left duct wall) with higher values of nanoparticle volume fraction, $\phi$. The skin friction $\frac{\partial w}{\partial y}$ at $y=1$ (at the right duct wall) again achieves the optimal value for silver and minimal value for $\mathrm{TiO}_{2}$. However at this duct wall, skin friction decreases in magnitude with $\phi, \operatorname{Pr}, S r, S c$ whereas it is elevated with $G r_{T}, G r_{C}$ and $A$. The skin friction $\frac{\partial w}{\partial x}$ at $x=0$ and at $x=1$ is maximal (optimal) for $\mathrm{SiO}_{2}$ and minimal for diamond nanoparticles. Skin friction increases in magnitude with $G r_{T}, G r_{C}$, $D f, \operatorname{Pr}, S r, S c, A$ and decreases with $\phi$.

Table 3 shows that the Nusselt number (heat transfer rate) $\frac{\partial \theta}{\partial y}$ at $y=0,1$ is maximum for diamond nanoparticle and minimum for $\mathrm{SiO}_{2}$ nanoparticles. An elevation in $\mathrm{Gr}_{T}$, $G r_{C}, D f, S r, S c, A$ does not significantly influence the rate of heat transfer. A rise in solid volume fraction strongly boosts the rate of heat transfer substantially at $y=0$ and at $y=1$. Heat transfer rate of regular fluid $(\phi=0)$ and nanofluid $(\phi \neq 0)$ are again significantly different - clear fluid (water) achieves much lower Nusselt numbers relative to water-based nanofluids. As the concentration of the suspended nanoparticles $\phi$ gradually elevates, the transfer of heat to the walls is accentuated. This characteristic of the fluid is the thermal conductivity which is enhanced greatly by increasing the concentration of the nanoparticles. The heat transfer rate is also elevated with Prandtl number, since temperatures in the bulk fluid are depressed and heat migrates more efficiently towards the duct walls. 
Table 4 indicates that the value of Sherwood number $\frac{\partial c}{\partial y}$ at $y=0,1$ is not hugely modified by the type of nanoparticle, $G r_{T}$ or $G r_{C}$ whereas it is slightly influenced with increment in $\phi, D f, \operatorname{Pr}, S r, S c, A . \frac{\partial c}{\partial y}$ at $y=0,1$ (left and right duct wall Sherwood numbers) increases as $\phi$ increases whereas it decreases with $D f, \operatorname{Pr}, S r, S c$, A. All the computations are carried out using $G r_{T}=10, G r_{C}=5, \phi=0.05, D f=1.0, \operatorname{Pr}=7.56, S r=2.0, S c=5.0, A=1 \quad$ unless otherwise indicated.

\section{CONCLUSIONS}

Motivated by deploying nanofluids in thermal duct optimization, in the present article, a theoretical study has been conducted for the influence of nanoparticles on natural convection inside a vertical rectangular duct with water as the base fluid and copper, diamond, $\mathrm{SiO}_{2}$ or $\mathrm{TiO}_{2}$ nanoparticles is studied in the presence of significant cross diffusion effects. The Tiwari-Das, Brinkman and Maxwell models have been deployed for nanoparticle-modified material characteristics e. g. thermal conductivity, viscosity. The transformed dimensionless partial differential equations for mass, momentum, energy and nanoparticle species conservation are solved with an efficient finite difference technique featuring a Southwell-Over-Relaxation (SOR) procedure. A grid-independence study is conducted. The influence of thermal and solutal Grashof number, solid volume fraction, Soret, Dufour, Schemidt, Prandtl numbers and aspect ratio on velocity, temperature, nanoparticle concentration, left and right duct wall skin friction, Nusselt number and Sherwood numbers are examined in detail. The principal conclusions which may be drawn from this parametric study can be summarized as follows:

1. Velocity of nanofluid is progressively decreased with silver, copper, diamond, $\mathrm{TiO}_{2}$ and $\mathrm{SiO}_{2}$ respectively.

2. The temperature is optimal for silver, diamond, copper and minimal for $\mathrm{TiO}_{2}$ and $\mathrm{SiO}_{2}$ nanoparticles, respectively. 
3. The nanoparticle concentration magnitude decreases sequentially for $\mathrm{SiO}_{2}, \mathrm{TiO}_{2}$, copper, diamond and silver, respectively.

4. The velocity field is enhanced with increment in thermal Grashof number and concentration Grashof number whereas the flow is suppressed with higher solid volume fraction, Dufour, Schmidt, Soret and Prandtl numbers.

5. Increasing aspect ratio serves to flatten the velocity profiles.

6. The temperature and concentration contours are not greatly influenced by nanoparticle type or other dimensionless parameters. When visualized locally at $y=0.5$ varying $x$ from 0 to 1 , the thermal profiles are not deflected with thermal Grashof number, Dufour parameter, Soret number, Schmidt number and aspect ratio. The concentration profiles are not altered with thermal Grashof number and aspect ratio whereas they contract with increment in concentration Grashof number, solid volume fraction and are amplified with an increment in Dufour, Prandtl, Soret and Schmidt numbers.

7. The skin friction $\frac{\partial w}{\partial y}$ at left and right walls achieves an optimal value for silver and minimal value for $\mathrm{TiO}_{2}$ nanoparticles. The shear stress $\frac{\partial w}{\partial x}$ at both duct walls is optimal for $\mathrm{SiO}_{2}$ and minimal for diamond nanoparticles.

8. The Nusselt number (heat transfer rate) at both duct walls is a maximum for diamond nanoparticles and minimal for $\mathrm{SiO}_{2}$ nanoparticles. Further the rate of heat transfer for the regular base fluid (water) is considerably lower than that for nanofluid.

9. The Sherwood number (mass transfer rate at both duct walls) is not substantially altered using any particular nanoparticle or with increment in thermal and concentration Grashof number; however, it is noticeably elevated with solid volume fraction and reduced markedly with Dufour, Prandtl, Soret, Schmidt number and duct aspect ratio.

The present study has revealed some interesting characteristics of thermal duct nanofluid transport in free convection for a variety of metallic/carbon-based nanoparticles. 
However, it has assumed rigid duct walls and that the nanofluid is Newtonian and electrically non-conducting. Some possible future pathways may be to incorporate biologically inspired duct walls (e. g. ciliated) and the deployment of magnetic nanofluids with rheological (e.g. viscoplastic) material behavior [55]. Efforts in this direction are currently underway.

\section{The authors do not have any conflict of interest}

\section{REFERENCES}

1. S.V. Patankar, Computation of Conduction and Duct Flow Heat Transfer, CRC Press, Florida (2019).

2. Barsi S, Moder J and Kassemi M. Numerical Investigation of LO2 and LCH4 Storage Tanks on the Lunar Surface. $\quad 44^{\text {th }}$ AIAA/ASME/SAE/ ASEE Joint Propulsion Conference and Exhibit, AAIA Paper 2008- 4749, 2008.

3. Godson L, Raja B, Mohan Lal D and Wongwises S. Enhancement of heat transfer using nanofluids -an overview. Renewable and Sustainable Energy Reviews 2010; 14: 629-641.

4. Choi SUS and Eastman JA. Enhancing thermal conductivity of fluids with nanoparticles. ASME International Mechanical Engineering Congress and Exposition, San Francisco, November 1995; 231: 99-106.

5. Murshed SMS, Leong $\mathrm{KC}$ and Yang $\mathrm{C}$. Enhanced thermal conductivity of $\mathrm{TiO}_{2}$ water based nanofluids. Int J Therm Sci 2005; 44: 367-373.

6. Zhang X, Gu H and Fujii M. Effective thermal conductivity and thermal diffusivity of nanofluids containing spherical and cylindrical nanoparticles. Exp Therm Fluid Sci 2007; 31: 593-599.

7. Ganguly S, Sikdar S and Basu S. Experimental investigation of the effective electrical conductivity of aluminum oxide nanofluids. Powder Technology 2009; 196: 326-330.

8. Sheikholeslami M and Ganji DD. Heat transfer of $\mathrm{Cu}$-water nanofluid flow between parallel plates. Powder Technology 2013; 235: 873-879.

9. Li N, Zeng Y, Liu Z, Zhong X and Chen S. Nanofluids containing Stearic Acid-Modified $\mathrm{CuO}$ Nanorods and their thermal conductivity enhancements. 
Nanoscience and Nanotechnology Letters 2015; 7: 314-317.

10. Song SL, Lee JH, Chang SH. CHF enhancement of SiC nanofluid in pool boiling experiment. Exp Therm Fluid Sci 2014; 52: 12-18.

11. Minkowycz WJ, Sparrow EM and Abraham JP. Natural convection in nanofluids, in: Nanoparticle Heat Transfer and Fluid Flow CRC Press 2012; 277317.

12. Einstein A. Eine neue Bestimmung der Molekuldimension. Ann Phys 1906; 19 289-306.

13. Brinkman HC. The viscosity of concentrated suspensions and solutions. J Chem Phys 1952; 20: 571-581.

14. Chen H, Ding Y, He Y and Tan C. Rheological behavior of ethylene-glycol based titania nanofluids. Chem Phys Lett 2007; 444: 333-337.

15. Murshed SMS, Leong KC and Yang C. A combined model for the effective thermal conductivity of nanofluids. Appl Therm Eng 2009; 29: 2477-2483.

16. Corcione M. Empirical correlating equations for predicting theeffective thermal conductivity and dynamic viscosity of nanofluids. Energy Conservation Management 2011; 52: 789-793.

17. Buongiorno J. A non-homogeneous equilibrium model for convective transport in flowing nanofluid. ASME J Heat Transfer 2005; 2: 599-607.

18. Tiwari AK and Das MK. Heat transfer augmentation in a two-sided lid-driven differentially heated square cavity utilizing nanofluids. Int J Heat Mass Transfer 2007; 50: 2002-2018.

19. Jou RY and Tzeng SC. Numerical research on natural convective heat transfer enhancement filled with nanofluids in rectangular enclosures. Int Commun Heat Mass Transfer 2006; 33: 727-736.

20. Umavathi JC and Shekar M. Effect of MHD on Jeffery-Hamel flow in nanofluids by Differential Transform method. Int. J. Eng Res Appl 2013; 3: 953-962.

21. Umavathi JC and Mohite MB. Convective transport in a porous medium layer saturated with a Maxwell nanofluid. J. King Saud University Engngn. Sciences 2016; 28: 56-68.

22. Selimefendigil F and Öztop H.F. Performance of TEG integrated channel with area 
expansion by using advanced passive techniques. Int. J. of Mechanical Sciences 2021; 194: 106210.

23. Selimefendigil F and Öztop H.F. Thermal management and modeling of forced convection and entropy generation in a vented cavity by simultaneous use of a curved porous layer and magnetic field. Entropy 2021; 23: 152 (18 pages).

24. Fontes DH, Santos DDOD, Padilla ELM and Filho EPB. Two numerical modeling of free convection heat transfer using nanofluids inside a square enclosure. Mech Res Comm 2015; 66: 34-43.

25. Kalidasan K, Velkennedy R and Kanna PR. Natural convection heat transfer enhancement using nanofluid and time-variant temperature on the square enclosure with diagonally constructed twin adiabatic blocks. Appl Therm Eng 2016; 92: 219-235.

26. Umavathi JC, Liu IC and Sheremet MA. Convective heat transfer in a vertical rectangular duct filled with a nanofluid. Heat Transfer Asian Research 2016; 45: 661-679.

27. Shekar M and Umavathi JC. Influence of viscous dissipation on non-Darcy mixed convection flow of nanofluid. Heat Transfer Asian Research 2017; 46: 176-199.

28. Hatami M. and Safari H. Effect of inside heated cylinder on the natural convection heat transfer of nanofluids in a wavy-wall enclosure. Int J Heat Mass Transfer 2016; 103: 1053-1057.

29. Al-Weheibi SM, Rahman MM, Alam MS and Vajravelu K. Nanofluids and the enhancement of natural convection heat transfer. Int J Mech Sci 2017; 131: 599-612.

30. Khanafer K, Vafai $\mathrm{K}$ and Lightstone M. Buoyancy-driven heat transfer enhancement in a two-dimensional enclosure utilizing nanofluids. Int. J. Heat and Mass Transfer 2003; 46: 3639-3653.

31. Ho CJ, Chen MW and Li ZW. Numerical simulation of natural convection of nanofluid in a square enclosure: effects due to uncertainties of viscosity and thermal conductivity. Int J Heat and Mass Transfer 2008; 51: 4506-4516.

32. Santra AK, Sen $\mathrm{S}$ and Chakraborty N. Study of heat transfer augmentation in 
a differentially heated square cavity using copper-water nanofluid. Int. J. Thermal Sci 2008; 47: 1113-1122.

33. Selimefendigil F and Öztop H.F. Performance assessment of a thermoelectric module by using rotating circular cylinders and nanofluids in the channel flow for renewable energy applications. Journal of Cleaner Production 2021; 279: 123426.

34. Selimefendigil F and Öztop H.F. Identification of pulsating flow effects with CNT nanoparticles on the performance enhancements of thermoelectric generator (TEG) module in renewable energy applications. Renewable Energy 2020; 162: 1076-1086.

35. Selimefendigil $F$ and Öztop H.F. MHD pulsating forced convection of nanoßuid over parallel plates with blocks in a channel. Int. J. Mechanical Sciences 2019; 157: 726-740.

36. Umavathi JC, Ojjela $\mathrm{O}$ and Vajravelu K. Numerical analysis of natural convective flow and heat transfer of nanofluids in a vertical rectangular duct using Darcy-Forchheimer-Brinkman model. Int J Thermal Sciences 2017; 11 511-524.

37. Umavathi JC. Rayleigh-Benard convection subject to time dependent wall temperature in a porous medium layer saturated by a Nanofluid. Meccanica 2015; 50: 981-994.

38. Diglio G, Roselli C, Sasso M and Umavathi JC. Borehole heat exchanger with nanofluids as heat carrier. Geothermics 2018; 72: 112-123.

39. Alsabery AI, Selimefendigi F, Hashim I, Chamkha AJ and Ghalambaz M, Fluidstructure interaction analysis of entropy generation and mixed convection inside a cavity with flexible right wall and heated rotating cylinder. International Journal of Heat and Mass Transfer 2019; 140: 331-345.

40. Bhatti MM and Abdelsalam SI. Bio-inspired peristaltic propulsion of hybrid nanofluid flow with Tantalum (Ta) and Gold ( $\mathrm{Au}$ ) nanoparticles under magnetic effects. Waves in Random and Complex Media, 2021;

DOI: $10.1080 / 17455030.2021 .1998728$

41. Khan SU, Al-Khaled K and Bhatti MM. Bioconvection analysis for flow of Oldroyd-B nanofluid configured by a convectively heated surface with partial slip effects. Surfaces and Interfaces 2021; 23: 100982 
42. Bhatti MM, Arain MB, Zeeshan A, Ellahi R and Doranehgard MH, Swimming of Gyrotactic microorganism in MHD Williamson nanofluid flow between rotating circular plates embedded in porous medium: Application of thermal energy storage J. of Energy Storage 2021;103511 https://doi.org/10.1016/j.est.2021.103511

43. Mojtabi A and Charrier-Mojtabi MC. Double-diffusive convection in porous media, in: Kambiz Vafai (Ed.) Handbook of Porous Media, Dekker, New York, 2000.

44. Gebhart B. Heat Conduction and Mass Diffusion, International Edition, MacGrawHill, New York, 1993.

45. Bég OA, Bakier AY and Prasad VR. Numerical study of free convection magnetohydrodynamic heat and mass transfer from a stretching surface to a saturated porous medium with Soret and Dufour effects. Computational Materials Science 2009; 46: 57-65.

46. Bég OA, Bég TA, Karim I, Khan MS, Alam MM, Ferdows M and Shamshuddin M. Numerical study of magneto-convective heat and mass transfer from inclined surface with Soret diffusion and heat generation effects: A model for ocean magnetohydrodynamic energy generator fluid dynamics. Chinese J Physics 2019; 60: 167-179.

47. Umavathi JC and Mohite MB. Double diffusive convective transport in a nanofluid-saturated porous layer with cross diffusion and variation of viscosity and conductivity. Heat Transfer Asian Research 2014; 43: 628-652.

48. Bhatti MM, Khalique CM, Bég T, Bég OA and Kadir A. Numerical study of slip and radiative effects on magnetic $\mathrm{Fe}_{3} \mathrm{O}_{4}$-water-based nanofluid flow from a nonlinear stretching sheet in porous media with Soret and Dufour diffusion. Modern Physics Letters B 2020; 33: 2050026 (24 pages).

49. Garvandha M, Narla VK, Tripathi D and Bég OA. Modelling the impact of melting and nonlinear radiation on nanofluid boundary layer flow over an inclined stretching cylinder with Buongiorno's model including cross diffusion and curvature effects, Applications in Nanotechnology, Chapter 4, Energy Systems and Nanotechnology”, "Advances in Sustainability Science and Technology" Book Series, SpringerGermany, August (2021). 
50. Umavathi JC and Sheremet MA. Onset of double-diffusive convection of a sparsely packed micropolar fluid in a porous medium layer saturated with a nanofluid, Microfluidic Nanofluidics 2017; 21: 121-128.

51. Pakravan HA and Yaghoubi M. Combined thermophoresis, Brownian motion and Dufour effects on natural convection of nanofluids. Int J Therm Sci 2011; 50: 394-402.

52. Vajjha RS and Das DK. Experimental determination of thermal conductivity of three nanofluids and development of new correlations. Int J Heat Mass Transfer 2009; 52: 4675-4682.

53. Mawell J. A Treatise on Electricity and Magnetism, $2^{\text {nd }}$ Edition, Oxford University Press, UK, 1904.

54. Hoffman JD. Numerical Methods for Engineers and Scientists, MacGaw-Hill, New York, 1992.

55. Akbar NS, Tripathi D, Bég OA and Khan ZH. MHD dissipative flow and heat transfer of Casson fluids due to metachronal wave propulsion of beating cilia with thermal and velocity slip effects under an oblique magnetic field. Acta Astronautica 128, 1-12 (2016). 